\title{
A Probabilistic Strategy for Understanding Action Selection
}

\author{
Byounghoon $\mathrm{Kim}^{1}$ and Michele A. Basso ${ }^{1,2}$ \\ Departments of ${ }^{1}$ Physiology and ${ }^{2}$ Ophthalmology and Visual Sciences, University of Wisconsin, Madison Medical School, Madison, Wisconsin 53706
}

Brain regions involved in transforming sensory signals into movement commands are the likely sites where decisions are formed. Once formed, a decision must be read out from the activity of populations of neurons to produce a choice of action. How this occurs remains unresolved. We recorded from four superior colliculus neurons simultaneously while monkeys performed a target selection task. We implemented three models to gain insight into the computational principles underlying population coding of action selection. We compared the population vector average (PVA)/optimal linear estimator (OLE) and winner-takes-all (WTA) models and a Bayesian model, maximum a posteriori estimate (MAP), to determine which predicted choices most often. The probabilistic model predicted more trials correctly than both the WTA and the PVA. The MAP model predicted $81.88 \%$, whereas WTA predicted $71.11 \%$ and PVA/OLE predicted the least number of trials at 55.71 and $69.47 \%$. Recovering MAP estimates using simulated, nonuniform priors that correlated with monkeys' choice performance, improved the accuracy of the model by $2.88 \%$. A dynamic analysis revealed that the MAP estimate evolved over time and the posterior probability of the saccade choice reached a maximum at the time of the saccade. MAP estimates also scaled with choice performance accuracy. Although there was overlap in the prediction abilities of all the models, we conclude that movement choice from populations of neurons may be best understood by considering frameworks based on probability.

\section{Introduction}

How perceptions, thoughts, decisions, and actions arise from the activity of populations of neurons is arguably the most vexing question in cognitive neuroscience. A number of lines of evidence from experimental work in monkeys indicate that perceptual decisions leading to eye movements (saccades) evolve within sensorimotor centers of the brain such as the lateral intraparietal area (LIP), parietal reach region, frontal eye field (FEF), and the superior colliculus (SC) in the midbrain (Gold and Shadlen, 2000; Roitman and Shadlen, 2002; Ratcliff et al., 2003, 2007; Horwitz et al., 2004; Scherberger and Andersen, 2007; Kim and Basso, 2008). A critical, unresolved issue is how the activity of neurons signaling targets and distractors is combined to contribute to a choice and then, how the combined activity is read out to result in a saccade. In other words, a key question remains unknown, what is the read-out rule that underlies movement choice?

Simultaneous recordings from multiple neurons within the monkey SC made during performance of a task in which one differently colored target appears in an array with three same-colored stimuli reveal that when the discriminability between the level of target and distractor neuronal activity is high, saccade choices are likely to be accurate. In contrast, when the discriminability be-

\footnotetext{
Received April 9, 2009; revised Dec. 26, 2009; accepted Dec. 31, 2009.

This work was supported by National Institutes of Health Grant EY13692 (M.A.B.). We also acknowledge the support of National Center for Research Resources Grant P51 RR000167 to the Wisconsin National Primate Research Center. We are especially grateful for invaluable discussions with Drs. Alexander Pouget, Anthony Movshon, and Lance Optican. We thank Drs. Merhdad Jazayeri, Alexander Grunewald, and Jochen Ditterich for comments on previous aspects of this work, Dr. Emilio Salinas for sharing Matlab code for computing the OLE, and the two anonymous reviewers for their helpful critiques.

Correspondence should be addressed to Dr. Michele A. Basso, Department of Physiology, University of Wisconsin, Madison, Medical School, 1300 University Avenue, Room 127 SM1, Madison, WI 53706. E-mail: michele@physiology.wisc.edu.

D0I:10.1523/JNEUROSCI.1730-09.2010

Copyright $\odot 2010$ the authors $\quad 0270-6474 / 10 / 302340-16 \$ 15.00 / 0$
}

tween the level of activity of target and distractor neurons is reduced, choice performance is likely to be poor. This result is consistent with the suggestion that SC neuronal activity signals an eye movement decision. It also reveals that the choice of which eye movement to make depends on the combined activity of neurons representing targets and distractors.

Because SC neurons are tuned broadly for target locations and saccade endpoints (Schiller and Koerner, 1971; Wurtz and Goldberg, 1972; Sparks, 1975, 1978; McIlwain, 1986; McIlwain, 1991), it is believed that the activity of large numbers of SC neurons are pooled to compute a vector average, which determines the saccade direction (Ottes et al., 1986; Van Gisbergen et al., 1987; McIlwain, 1991; Groh, 2001) in much the same way as arm movement directions are coded by motor cortex neurons (Georgopoulos et al., 1986; Schwartz et al., 1988). Indeed, simultaneous electrical activation of two regions of the SC results in saccades with vectors that are averages of the saccade vectors produced by stimulation of each site independently (Robinson, 1972). Furthermore, inactivation of regions of the SC produces inaccuracies in saccade directions and lengths that are largely consistent with predictions of a population vector averaging (Lee et al., 1988; Quaia et al., 1998; Hanes and Wurtz, 2001). Whereas these experiments relied on measures of saccades made to single spots of light, more recent experiments requiring the identification of one target from an array of distractor stimuli (Basso and Wurtz, 1998; McPeek and Keller, 2004) or choosing between two simultaneously or sequentially appearing stimuli (Port and Wurtz, 2003; Li and Basso, 2005; Kim and Basso, 2008) suggest that winner-takes-all or probabilistic strategies may more accurately reflect the information in SC neuronal populations.

Based on our previous work and that of others showing that SC neuronal activity scales with the likelihood of a correct saccade choice (Basso and Wurtz, 1998; Dorris and Munoz, 1998; Kim 
and Basso, 2008), here we explored whether SC neurons could formally encode information about saccade choices probabilistically. We implemented three different models to reveal principles underlying how SC neuronal activity might be encoded by the population and then interpreted by downstream structures during the performance of a target selection task. Unique to our experiments is that we recorded from four SC neurons simultaneously and each neuron contained one element of the visual display within its response field (RF). We determined the best estimate of the saccade choice by implementing a probabilistic (Bayesian) model, the maximum a posteriori estimate (MAP), and winner-takes-all (WTA) and population vector average (PVA)/optimal linear estimator (OLE) models. To assess the models, we compared how well each estimate predicted the saccade choice on a trial-by-trial basis for correct and error trials. Furthermore, we examined for the first time the temporal development of the maximum a posteriori estimate. We found that the MAP model provided the best estimate of saccade choices across all trials, took time to develop, and scaled with the monkeys' choice performance. These results are consistent with a probabilistic coding strategy underlying movement choice.

\section{Materials and Methods}

\section{Physiological and eye movement monitoring procedures}

For electrophysiological recording of SC neurons and monitoring eye movements, cylinders and eye loops were implanted in two rhesus monkeys (Macaca mulatta) using documented procedures (Judge et al., 1980; Kim and Basso, 2008; Li and Basso, 2008). We recorded from 120 neurons within the intermediate layers of the SC. We used a subset of the same dataset used for a previous report (Kim and Basso, 2008). Neurons were recorded simultaneously in sets of four. In monkey $\mathrm{m}$, we recorded 13 sets of four SC neurons $(n=52)$. In monkey c, we recorded 17 sets of four SC neurons $(n=68)$. Of the total 120 neurons in both monkeys, all neurons were defined statistically as buildup/prelude (Munoz and Wurtz, 1995; Basso and Wurtz, 1998; McPeek and Keller, 2002; Li and Basso, 2005, 2008) except three, which were defined statistically as visual-tonic (McPeek and Keller, 2002; Li and Basso, 2008).

Neurons were recorded with four independently moveable, tungsten microelectrodes (Frederick Haer) with impedances between 0.3 and 1.0 $\mathrm{M} \Omega$ measured at $1 \mathrm{kHz}$. Four electrodes were aimed at the SC, each through different stainless steel guide tubes held in place by a plastic grid secured to the cylinder (Crist et al., 1988). Two were aimed at one SC, and two were aimed at the other SC. Electrodes were introduced independently, and neurons (action potential waveforms) were isolated on each electrode sequentially. RFs of SC neurons were mapped online. Mapping was done by moving a spot around the screen and having monkeys make saccades to the different spots. We listened for maximal discharge and also monitored raster plots of the discharge online. We considered the center of the RF to be the location at which a saccade was associated with maximal discharge of the neuron (audibly and visually). When recorded in the single target condition, we ensured that each stimulus drove only one of the recorded neurons. In other words, the RFs of each of the four neurons were nonoverlapping when recorded in the single target condition. Action potential waveforms were filtered and amplified by a differential amplifier (Alpha Omega; MCP-Plus) and then sampled and digitized (Measurement Computing; PCI-DAS4020/16). The digitized waveforms were identified and sorted with an interactive computer program (Mex; National Eye Institute) allowing the experimenter to sort waveforms in real time. Neuronal data were also saved to disk as waveforms and sorted offline to confirm the adequacy of the online discrimination. For offline analysis, we used custom software (written and compiled in Delphi 5.0) that sorted spikes based on time-voltage criteria. Using the magnetic induction technique (CNC Engineering) (Fuchs and Robinson, 1966), voltage signals proportional to horizontal and vertical components of eye position were filtered ( 8 pole Bessel $-3 \mathrm{~dB}, 180 \mathrm{~Hz}$ ), digitized at 16-bit resolution, and sampled at $1 \mathrm{kHz}$ (National Instruments; PCI-6036E). The data were saved for offline analysis using an interactive computer program (Dex, National Eye Institute) designed to display and measure eye position and calculate eye velocity. We used an automated procedure to define saccadic eye movements by applying velocity and acceleration criteria of $20 \% \mathrm{~s}$ and $8000 \% \mathrm{~s}^{2}$, respectively. The adequacy of the algorithm was verified and adjusted as necessary on a trial-by-trial basis by the experimenter.

All experimental protocols were approved by the University of Wisconsin-Madison Institutional Animal Care and Use Committee and complied with and generally exceeded the standards set by the Public Health Service policy on the humane care and use of laboratory animals.

\section{Behavioral procedures}

We used a real-time experimental data acquisition and visual stimulus generation system Rex, Vex, and Mex, developed and distributed by the Laboratory of Sensorimotor Research National Eye Institute (Hays et al., 1982) to create the behavioral paradigm and acquire two channels of eye position and four channels of neuronal data. Trained monkeys sat in a custom primate chair with head stabilized during the experimental session (typically $3-5 \mathrm{~h}$ ). Visual stimuli were rear-projected onto a screen at $51 \mathrm{~cm}$ distance from the subject using a projector (LP130, Infocus) with a native resolution of $1024 \times 768$ and operating at $60 \mathrm{~Hz}$. A photocell secured to the screen sent a transistor-transistor logic pulse to the experimental PC, providing an accurate measure of stimulus onset. The fixation spot at the center of the screen had a (mean of three measurements) luminance of $1.52 \mathrm{~cd} / \mathrm{m}^{2}$. Visual stimuli each had luminance values of 5.8 $\mathrm{cd} / \mathrm{m}^{2}$ (mean of three measurements). The background luminance was $0.58 \mathrm{~cd} / \mathrm{m}^{2}$ (mean of three measurements). The PC for the visual stimulus display was a slave device to the PC used for experimental control and data acquisition.

After fixating on a centrally located spot $\left(0.6^{\circ}\right.$ diameter $)$ for a random time of $1800-2300 \mathrm{~ms}$, four spots $\left(1.0^{\circ}\right.$ diameter $)$ appeared and the central spot disappeared. Each spot was located in the center of each empirically defined RF of the four SC neurons (Fig. 1a) (see text above). The task required monkeys to choose the differently colored target within $\sim 300$ ms by making a saccade to the differently colored spot immediately after the disappearance of the fixation spot (coincident with the array onset). The target could be either red among green distractors or green among red distractors. The color arrangement of the display was fixed each day of recording but varied across recording days. After making a choice, monkeys maintained fixation at the target spot for a random time of 500-600 $\mathrm{ms}$ and then received fluid reward. The location of the target spot was randomized (with replacement) among the four possible locations. On interleaved trials, a single spot appeared in each of the four possible locations. Two spots appeared in each hemifield although the exact location of the visual spots depended upon the location of the four electrodes within the SC (Fig. 1b).

\section{Data analysis}

We implemented three broad classes of model to recover the population estimate of saccade choices in the selection task. The simplest model was a WTA (Feldman, 1982). Next was a PVA model similar to that implemented by Georgopoulos et al. (1986) in motor cortex and Port and Wurtz (2003) in SC. We also implemented an optimized vector averaging method developed by Salinas and Abbott (1994) referred to as the OLE. The final model we implemented was a likelihood estimator based on Bayesian inferential statistics, the MAP. This model arises from ideas formulated previously (Sanger, 1996, 2002, 2003; Oram et al., 1998) and recently extended to sensory processing in the middle temporal area (MT) (Jazayeri and Movshon, 2006) and decision making in LIP (Beck et al., 2008). Our implementation of this model is similar but has important extensions of this recent work that are discussed below. In the text that follows, we describe how each model was implemented. We assessed the quality of the model prediction by comparing the saccade choice recovered by the model to the actual saccade choice monkeys made, regardless of whether the trial was correct or in error. 

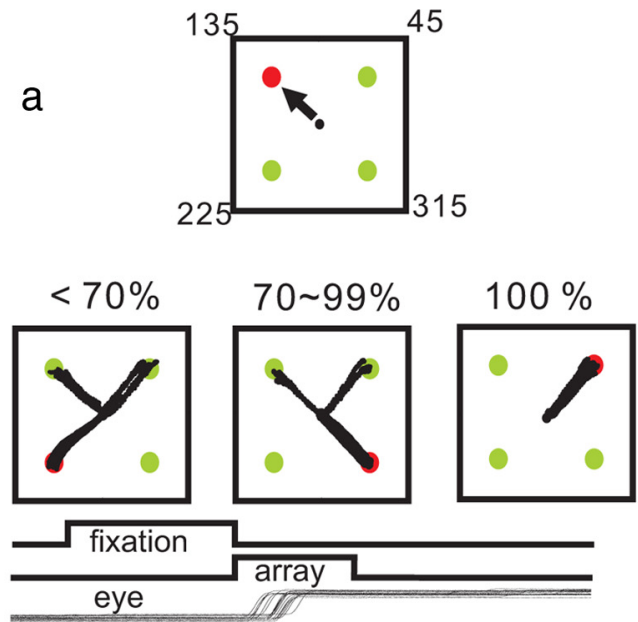

b

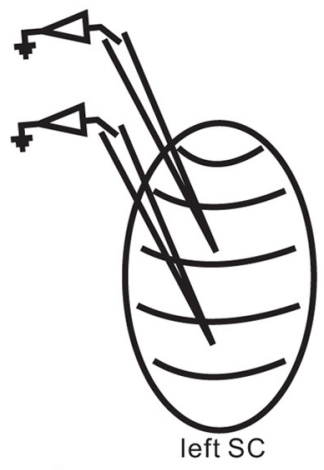

C
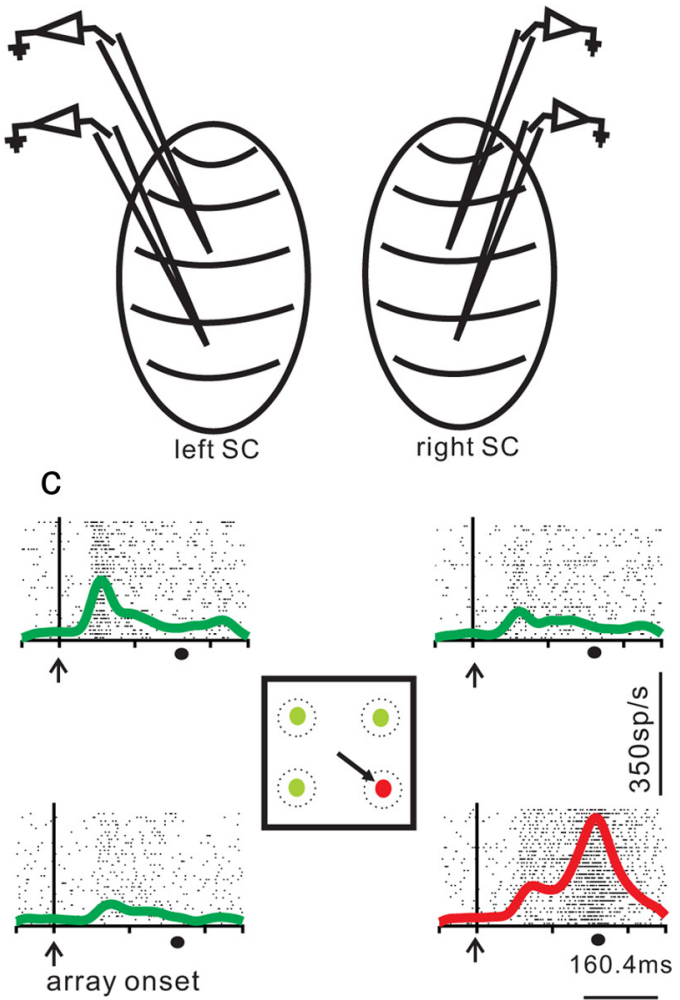

$\uparrow$

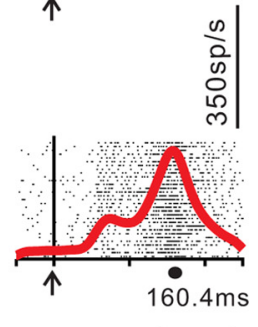

$\overline{100 \mathrm{~ms}}$

Figure 1. Saccade choices in a target selection task. $\boldsymbol{a}$, Each square shows the spatial arrangement of the task. The spot that is colored differently indicates the target, whereas the same colored spots indicate distractors (either green among red or red among green). In this example, the target is red. The black circle in the center of each square indicates the location of the fixation spot. In the task, the array appeared and the fixation spot disappeared simultaneously, cueing monkeys to make a saccade to the target (black arrow). The black lines in each of the three bottom squares show actual saccade trajectories. Each of the three squares show examples of choice performance of $<70 \%, 70-99 \%$, and $100 \%$ correct. Note that all spots were normalized to $45^{\circ}, 135^{\circ}, 225^{\circ}$, and $315^{\circ}$ positions for display. The temporal sequence of the task appears as labeled, black lines below the spatial depiction. $\boldsymbol{b}$, Schematics of the two SCs and the electrodes for multiple neuron recordings. $c$, Examples of four neurons recorded simultaneously from the SC. Each tick in a row indicates the time of occurrence of an action potential, and each row indicates a trial. The four raster plots correspond to the neurons with RF centers located at the positions shown in the center square. The spike density function (SDF; $\sigma=10$ $\mathrm{ms}$ ) is superimposed on the raster plots. Green lines show SDFs for distractor neurons, and red lines show SDFs for target neurons. The mean reaction time of the saccade is shown by the black circle at the bottom of the traces labeled $160.4 \mathrm{~ms}$. Each trace is aligned to the onset of the array, marked by the upward arrow and vertical line and labeled "array onset."

Bayesian model: MAP. To compute the posterior probability distribution over the four possible saccade choices, we first consider Bayes' rule, which states the following:

$$
P(s \mid r)=\frac{P(r \mid s) P(s)}{P(r)} .
$$

$P(s \mid r)$ is the conditional probability of observing a particular saccade choice given a particular discharge rate, also called the posterior. $P(r \mid s)$ is the conditional probability of observing a particular discharge rate when a particular saccade occurs. This value is known as the likelihood. $P(s)$ is the probability of a saccade choice or the prior. $P(r)$ is the probability of a particular discharge rate in spikes per second. A Bayesian framework provides a way to quantify guesses about events when faced with uncertainty. The probabilities in Bayes' rule indicate the strength of a belief from 0 to 1 . Since the probability of the discharge rate $P(r)$ is independent of the saccade choice, we can restate the posterior as proportional to the product of the likelihood and the prior (Földiak, 1993; Oram et al., 1998):

$$
P(s \mid r) \propto P(r \mid s) P(s) .
$$

In words, the probability of a saccade choice given the observation of a particular discharge $\{P(s \mid r)\}$ is proportional to the conditional probability of the discharge given the saccade choice $\{P(r \mid s)\}$ multiplied by the prior $\{P(s)\}$. Thus, the posterior is proportional to the product of the likelihood and the prior. Note that for display, we include the normalization factor $P(r)$ so that the scaling ranges from 0 to 1 and so that bona fide probabilities can be compared across conditions (Oram et al., 1998).

We implemented two prior $\{P(s)\}$ distributions. In one, we used a discrete uniform prior with four Dirac delta functions at each of four possible target choices:

$$
P(s)=0.25 \sum_{i=1}^{4} \delta(s-(i-1) \pi / 2) .
$$

$P(s)$ is a discrete prior distribution describing the four possible saccade target choices $(s)$ separated by $90^{\circ}$. The four delta functions $(\delta)$ for each choice are defined by shifts from the first by $90^{\circ}(\pi / 2)$. We summed the four delta functions and multiplied by 0.25 (four possible choices) so the prior distribution had uniform probabilities for the four possible target/saccade choices (Fig. $2 h$ ). This reflects the experimental situation used. Each of the four possible saccade choices represents one of the four possible target locations and each of these occurred with an equal $(25 \%)$ probability.

For the second implementation, we used a simulated $P(s)$ and determined the distribution that maximized the MAP model's performance. To do this, we generated four random values that summed to 1.0 to simulate probability distributions:

$$
P(s)=\sum_{i=1}^{4}\left[\operatorname{rand}_{i} / \sum_{j=1}^{4} \operatorname{rand}_{j}\right] \delta(s-(i-1) \pi / 2) .
$$

Equation 4 defines a discrete prior distribution as a summation of four delta functions as in Equation 3. Each value in Equation $4\left(\operatorname{rand}_{1-4}\right)$ varied independently from 0.01 to 0.97 with an interval of 0.01 . To ensure that the sum of this distribution was 1.0, we divided each of four random values $\left(\mathrm{rand}_{i}\right)$ by the sum of all values $\left(\sum_{j=1}^{4} \mathrm{rand}_{j}\right)$. This was then multiplied by the $90^{\circ}$ shifted delta functions yielding a prior probability distribution that is a discrete, nonuniform distribution with four values, one for each of four possible saccade choices (Fig. 2i). Because they were generated randomly, Equation 4 produced the same combinations in some cases. Therefore we selected only the unique combinations. This left a total of 156,941 unique combinations of four values. With the simulated prior distributions in hand, we then recomputed the MAP estimate using each one of these 156,941 simulated prior distributions. We identified the simulated prior distribution that when used to recover the MAP estimate, resulted in the same or better prediction accuracy as the MAP estimate with the nonuniform prior distribution. The actual prior distribution that monkeys might use is unknowable. The prior distribution used, however, is likely to be related to the final choice behavior (distribution of saccade choices). To test this, we calculated the 


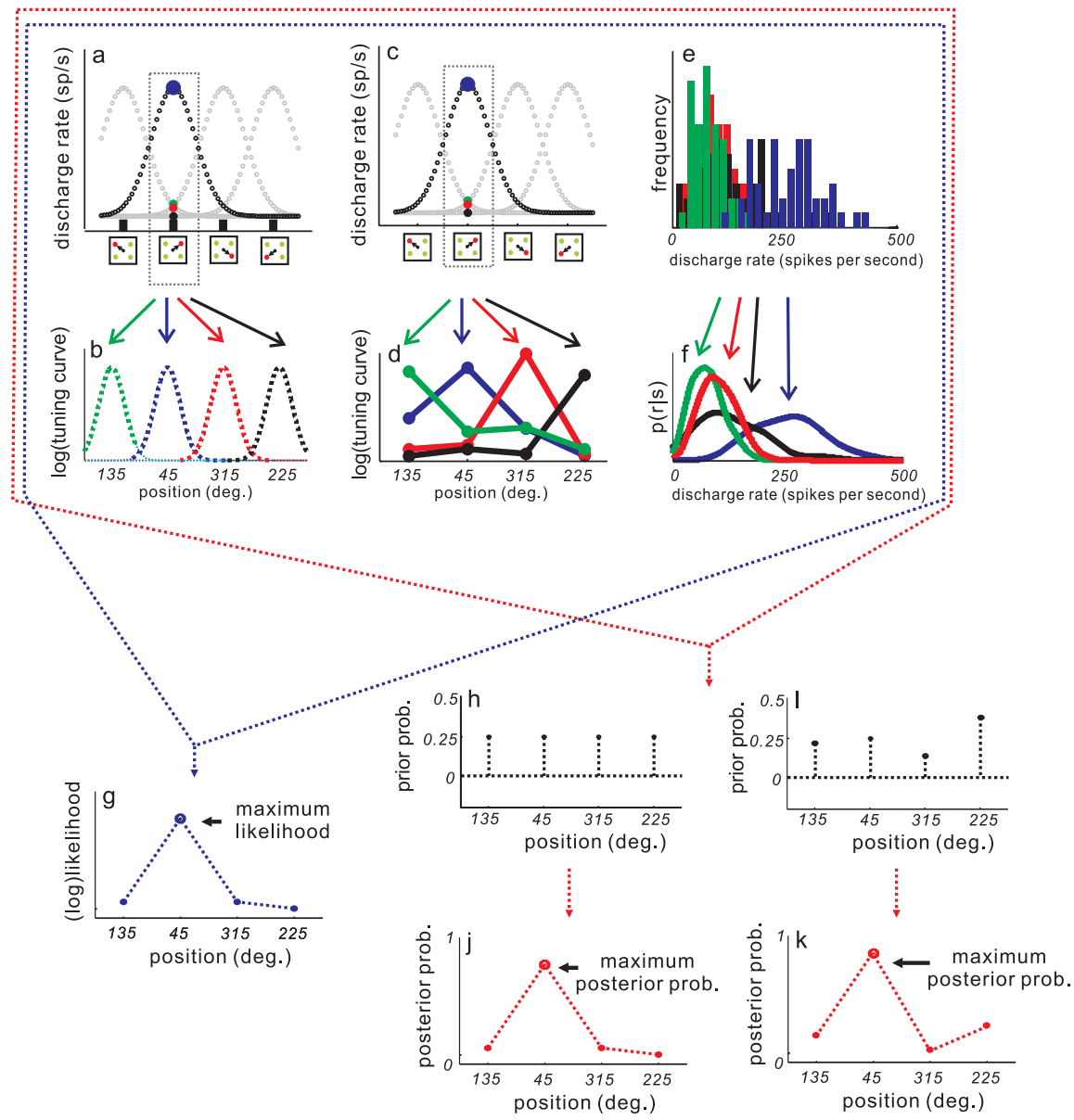

Figure 2. Computing the posterior distribution across saccade choices. $\boldsymbol{a}$, Simulated, idealized responses (in spikes per second) of four SC neurons in the four different target conditions of the task. Small squares on the abscissa show the four possible stimulus configurations of the task. The four curves simulate the activity of each neuron in each of the four possible conditions. For display, these data were simulated as Gaussian functions with $\sigma=40^{\circ}$. Each Gaussian peak was shifted by $90^{\circ}$ to represent the four possible locations. Each simulated response shows a peak discharge when the target corresponds to the preferred location for the neuron and shows a reduced level of discharge as the target moves away from the preferred location. The dotted rectangle around the black simulated response shows an example response whose peak discharge (blue circle) appears for targets located at $45^{\circ}$ and a reduced response for target locations elsewhere. The green dot, red dot, and black dot show the simulated discharge rate for the other three neurons when the target appears outside of their RF. $\boldsymbol{b}$, Simulated weights for $\mathrm{SC}$ neurons. Each tuning curve represents the probability of neuronal discharge given a stimulus position from 0 to $359^{\circ}$ (in steps of $0.1^{\circ}$ ). The tuning curves were simulated as Gaussian functions of equal amplitude (arbitrary units) and widths of $20.6^{\circ}$ (Edelman and Keller, 1998). The logs of these Gaussian functions were used as weights. The green curve shows the weight for the neurons with the highest discharge rate in the $135^{\circ}$ target condition. The blue curve shows the weight for the neurons with peak rates in the $45^{\circ}$ target condition. The red and cyan curves show weights for the neurons with peak rates in the $315^{\circ}$ and $225^{\circ}$ target conditions, respectively. c, The same as in $\boldsymbol{a}$ $\boldsymbol{d}$, Each point is the measured discharge from an $\mathrm{SC}$ neuron when monkeys made saccades to one of each of the four possible locations. $\boldsymbol{e}$, Frequency histogram of discharge rates measured from the sets of four neurons in the four possible target conditions across all datasets. The activity of target neurons is shown in the blue bars, whereas the activity of distractor neurons is shown in the green, red, and black bars. These histograms formed the basis for the density estimation (see Materials and Methods for details). $\boldsymbol{f}$, The result of the density estimation (smooth histograms in $\boldsymbol{e}$ - see Materials and Methods) is the likelihood of a response given prior probability distribution the result is a (log) likelihood distribution. $\boldsymbol{h}$, The uniform prior probability distribution. Each dot shows the probability of one saccade choice. Since the target could appear in each of the four locations with equal probability, the distribution is uniform. $\boldsymbol{i}$, Same as in $\boldsymbol{h}$ but using a simulated, nonuniform prior distribution (see Materials and Methods). $\boldsymbol{j}$, Estimated posterior probability distribution for one example target condition $\left(45^{\circ}\right)$. Multiplying all four conditional probability across the four neurons and the prior, results in the posterior probability for the example target condition across the four neurons. $\boldsymbol{k}$, Same as in $\boldsymbol{j}$ for the distribution using a nonuniform prior. a particular saccade choice. Distractor neurons are green, red, and black. The target neurons are blue. $\boldsymbol{g}$, Without incorporating a

A critical aspect of computing the MAP estimate is how to determine $P(r \mid s)$. In recent work, it was shown that a good characterization of $P(r \mid s)$ can be obtained by assuming a Poisson probability distribution or any distribution of the exponential family with linear sufficient statistics (Ma et al., 2006; Beck et al., 2007). So our first approach was to use a Poisson probability distribution constrained by the tuning properties of our SC neurons to estimate $P\left(r_{1-4} \mid s\right)$. Indeed as a first approximation, our neurons behaved in a linear sufficient manner as determined by assessing the relationship of the variance of action potential counts across trials to the mean of the action potential counts (see supplemental Fig. 1a, available at www.jneurosci.org as supplemental material). We used the Poisson probability density distribution (PDD) in place of the likelihood, $P\left(r_{1-4} \mid s\right)$, where $\lambda$ is the expected number of action potential occurrences in a Poisson probability distribution and in our implementation was the tuning curve $\left\{f_{i}(s)\right\}$ of the $i$ th neuron. The exponent and the denominator $\left(r_{i}\right)$ are the numbers of action potentials measured in a $20 \mathrm{~ms}$ time epoch $(28 \mathrm{~ms}$ before to 8 $\mathrm{ms}$ before the onset of the saccade):

$$
P\left(s \mid r_{1-4}\right) \propto\left[\prod_{i=1}^{4} \frac{f_{i}(s)^{r_{i}}}{r_{i} !} e^{-f_{i}(s)}\right] P(s) .
$$

The posterior probability of a saccade choice $(s)$ given the discharge of all four neurons $\left\{P\left(s \mid r_{1-4}\right)\right\}$ was estimated by computing the conditional probability for each of the four neurons and multiplying by the prior probability. To combine the neuronal activity linearly, we made the reasonable assumption that the neurons in our sample were statistically independent. We describe how we deal with this assumption in supplemental Figures $1 b$ and 2 (available at www.jneurosci.org as supplemental material) and in Results. To allow summation rather than multiplication, we took the logarithm of Equation 5:

$$
\begin{aligned}
\log \left(P\left(s \mid r_{1-4}\right)\right) & =\sum_{i=1}^{4} r_{i} \log \left(f_{i}(s)\right)-\sum_{i=1}^{4} f_{i}(s) \\
& -\sum_{i=1}^{4} \log \left(r_{i} !\right)+\log (P(s)) .
\end{aligned}
$$

$\sum_{i=1}^{4} \log \left(r_{i} !\right)$ can be ignored because it is independent of the saccade choice. However, we maintained the $\sum_{i=1}^{4} f_{i}(s)$ term because across our neurons, the tuning curves were different (Fig. 2d). As a result this term does not sum to a constant and must remain in the model. The calculation simplifies to the following:

$$
\begin{array}{r}
\log \left(P\left(s \mid r_{1-4}\right)\right)=\sum_{i=1}^{4} r_{i} \log \left(f_{i}(s)\right)-\sum_{i=1}^{4} f_{i}(s) \\
+\log (P(s)) .
\end{array}
$$

Note that for $f_{i}(s)$, we also implemented a verdistribution of saccade choices using the actual behavior of the monkeys on a trial-by-trial basis. In Figure $4 a$, we show through simulations of multiple possible prior distributions that there is a strong relationship between the simulated prior distribution and the distribution of saccade choices. These correlations validate our use of the simulated before recover the MAP estimate of the saccade choice. sion of the MAP model in which we used identical Gaussian functions (Edelman and Keller, 1998) peak shifted by $90^{\circ}$ to simulate SC tuning curves (Fig. $2 b$ ). In this case, the $\sum_{i=1}^{4} f_{i}(s)$ term was omitted from the model because summing over these functions is a constant. To avoid overestimation of the model, we implemented a leave-one-out cross- 
validation procedure. For this, we extracted one trial from each dataset and used the remaining trials to estimate $P(r \mid s)$ from each set. We then recovered the posterior from the extracted trial. This procedure was repeated for all trials for each dataset. Equation 7 returns one value for each of the possible saccade choices. Computing this value for each of the four possible saccade choices $\left(s_{j}\right)$, where $j=1-4$, defines the posterior distribution across the four possible saccade choices. In the case of the uniform prior distribution, the result is a Bayesian estimator that yields the same result as a maximum likelihood estimator as formalized by others (Sanger, 1996, 2002; Jazayeri and Movshon, 2006). In the case of the nonuniform prior, the result is a Bayesian estimator distinct from a maximum likelihood. To determine how well the posterior distribution predicted monkeys' actual choices, we compared the MAP with the saccade choice on a trial-by-trial basis:

$\operatorname{MAP}\left(s_{j}\right)=\arg \max \left(\log \left(P\left(s_{j} \mid r_{1-4}\right)\right)\right.$

$$
=\arg \max \left[\sum_{i=1}^{4} r_{i} \log \left(f_{i}\left(s_{j}\right)\right)+\log \left(P\left(s_{j}\right)\right)\right] .
$$

When the saccade choice and the maximum a posteriori estimate corresponded, we considered the model to have a correct prediction. Figure 2 provides a graphic depiction of the MAP model along with the different $P(r \mid s)$ and $P(s)$ implementations.

Determining the likelihood using a Poisson PDD relies on two assumptions (Földiak, 1993; Sanger, 1996, 2002; Oram et al., 1998; Jazayeri and Movshon, 2006; Ma et al., 2006; Beck et al., 2008). The first is that the occurrence of each action potential in a spike train is independent of the occurrence of other action potentials in the train. If time between successive action potentials is random, we can consider the train of action potentials as a Poisson process. A common way to assess whether discharge statistics can be described as a Poisson process is to determine the index of proportionality, also referred to as the Fano factor, which is the ratio of the variance of the number of action potentials in an epoch to the number of action potentials in an epoch across trials. On a linear plot, a slope of 1.0 indicates linearity. To determine the Fano factor of SC neurons, we counted the number of action potentials within the epoch 28 to $8 \mathrm{~ms}$ before the onset of a saccade for each trial in a dataset. We then determined the trial-to-trial variance of the action potential counts by subtracting individual trial counts from the mean count and squaring that quantity. This was done for the set of trials across all neurons. We then computed the mean of the difference measure and the mean of the action potential count and plotted these values for each neuron. Supplemental Figure $1 a$ (available at www.jneurosci.org as supplemental material) shows the action potential count variance against the mean count across all 120 SC neurons when the stimulus in the neurons' RF was either a target or a distractor. The Fano factor for neurons when targets were in their RFs was $1.44(n=120)$. The Fano factor for neurons when distractors were in their RFs was 1.03 $(n=360)$. These observations are consistent with the assumption of linear sufficient statistics, at least for distractor activity.

Supplemental Figure 1 (available at www.jneurosci.org as supplemental material) shows that when targets appeared in the RF, the variance to mean relationship diverged from linearity. This is because SC neurons are exhibiting rapid increases in discharge associated with the saccade to the target in the RF. To deal with this deviation from linearity, we extended our probabilistic model to eliminate the Poisson probability distribution to estimate $P\left(r_{1-4} \mid s\right)$. Instead, we determined $P\left(r_{1-4} \mid s\right)$ directly by using a nonparametric density estimation procedure (Optican and Richmond, 1987; Scott, 1992). Figure 2, $e$ and $f$, shows graphically how this was performed. Nonparametric density estimation is simply smoothing a frequency histogram. This procedure is similar to that used to calculate spike density functions from raster plots (MacPherson and Aldridge, 1979). We first plotted the distribution of discharge rates measured in the four possible target condition during the $20 \mathrm{~ms}$ epoch measured 28 to $8 \mathrm{~ms}$ before saccade onset. We applied a smoothing $\operatorname{kernel}(k[])$ :

$$
k[]=\frac{e^{(x-r)^{2 /-2 h^{2}}}}{\sqrt{2 \pi}} \cdot h,
$$

where $h$ is the number of bins, $r$ is the discharge rate measured in the 20 $\mathrm{ms}$ epoch, and the domain of $x$ is the set of all numbers defined by the discharge rate. From here, the Gaussians are summed over the discharge rates, and the sum is weighted by the number of bins in the frequency distribution $(n)$. Assuming a normal probability density, $h$ can be estimated by minimizing the (averaged) mean integrated squared error (AMISE) (Scott, 1992):

$$
h=\left(\frac{4}{3}\right)^{1 / 5} \sigma n^{-1 / 5} \approx 1.06 \sigma n^{-1 / 5} .
$$

Convolving the histograms with the smoothing kernel in Equation 9 yields the empirical probability density distribution:

$$
P(r \mid s)=\frac{1}{n} \sum_{i=1}^{n} k\left[\frac{x-r_{i}}{h}\right] .
$$

This procedure was done to obtain a PDD for each neuron. From here, we could extract the $P(r \mid s)$ directly to compute the posterior distribution over the four possible saccade choices again on a trial-by-trial basis:

$$
P\left(s \mid r_{1-4}\right)=\left[\prod_{i=1}^{4} P\left(r_{i} \mid s\right)\right] P(s) / P(r) .
$$

As in the model shown in Equation 8, when the saccade choice and the maximum a posteriori estimate determined from Equation 12 agreed, we considered the model to have a correct prediction.

The second assumption that is required to compute the posterior probability is that the noise correlations between the four neurons should be independent. Because we were careful to record from neurons with nonoverlapping RFs, we assumed independence of the neuronal discharge. We calculated the noise correlation coefficients between neuronal responses to confirm our assumption (Averbeck et al., 2006). Since we have four neurons, combining each into unique pairs resulted in six pairs allowing us to test all possible noise correlations between the four neurons $[$ total conditions $=6($ pairs $) \times 4($ target conditions $) \times 30($ datasets $)=720]$. Neuronal activity was measured 28 to $8 \mathrm{~ms}$ before saccade onset for all six pairs. Across our sample of 720 pairs, only 9.86\% (71/ 720 ) of the pairs had statistically significant noise correlations (supplemental Fig. 1b, available at www.jneurosci.org as supplemental material). To confirm that the noise correlations were accounted for in our model or did not contribute much to the result of the model, we performed a shuffled analysis of our data (as shown in supplemental Fig. 2, available at www.jneurosci.org as supplemental material).

It is important to note that although our implementation is Bayesian in the sense that we calculated a posterior probability by combining likelihoods and prior distributions, there are some differences between our model and true Bayesian estimator. First, the likelihood distributions in our model are discrete and are estimated from four individual neurons. Any additional variability that may be conveyed leading to a saccade choice is ignored. Second, the prior distributions we implemented are also discrete and deterministic. Third, and as noted above, since we cannot ever know the true prior distribution, we simulated it. As shown in Figure $4 a$, the simulated prior distribution correlates with the distribution of saccade choices made by the monkeys. This validates our approach and indicates that the simulated prior distribution we used to recover the MAP estimates of saccade choice was a good approximation to the actual prior used by the monkeys while performing this task.

WTA. We implemented a WTA model by computing the mean discharge rate during an interval $28 \mathrm{~ms}$ to $8 \mathrm{~ms}$ before the onset of a saccade (Miyashita and Hikosaka, 1996) on a trial-by-trial basis from each of the four neurons across all 30 sets. For each set of four neurons, the neuron with the highest discharge rate was defined as the "winner." We then compared the RF location of the winner neuron on each trial to the 
location of the saccade choice on that trial. Because there were four neurons, each representing one possible location, a correct prediction occurred when the neuron corresponding to the saccade choice had the highest discharge rate.

$P V A$. To compute the population vector average $\left(\vec{V}_{\text {population }}\right)$, we considered each of the four neurons simultaneously recorded as one of a larger population of neurons representing one of the possible saccade choices. We computed the $\vec{V}_{\text {population }}$ for each trial using the four neurons, one representing the target (target neuron) and the other three representing the distractors (distractor neurons). We implemented a similar procedure for computing the $\vec{V}_{\text {population }}$ as used previously in motor cortex (Georgopoulos et al., 1986) and SC (Port and Wurtz, 2003). However, we adopted a normalization procedure suggested by Salinas and Abbott (1994) to avoid obtaining negative vectors:

$$
\vec{V}_{\text {population }}=\frac{\sum_{i=1}^{4}\left(r_{i}\left(\sqrt{\sum_{j=1}^{4} r_{j}^{2}}\right)^{-1} \vec{S}_{i}\right)}{4} .
$$

We computed the neuronal population vector average using Equation 13 , where $r_{i}$ was the mean discharge rate for each $i$ th neuron measured during the $20 \mathrm{~ms}$ interval immediately before the onset of the saccade during the selection task ( $28 \mathrm{~ms}$ to $8 \mathrm{~ms}$ before saccade onset; note that this is the same interval as used for MAP and WTA). Since each neuron had different discharge rates and different baseline rates, it was necessary to normalize the neuronal responses to avoid arbitrary biases in $\vec{V}_{\text {population }}$. The normalized neuronal response was determined by calculating $r_{i}\left(\sqrt{\sum_{j=1}^{4} r_{j}^{2}}\right)^{-1}$. This term was then multiplied by the unit vector $\vec{S}_{i}$, which was defined as the average saccade direction determined by the actual eye movements made by the monkeys. This result was then summed and divided by four to obtain $\vec{V}_{\text {population }}$. The saccade choice with the smallest angular difference between it and the $\vec{V}_{\text {population }}$ was considered a correct model prediction. One assumption of this version of the PVA is that the SC contains a homogenous representation of all possible saccades. So we also implemented a model developed by Salinas and Abbott (1994) that optimizes the neuronal population vector to take into account inhomogeneous distributions. For this model, we determined $\vec{V}_{\text {population }}$ by summing the discharge rates weighted by an optimized saccade vector:

$$
\begin{gathered}
\vec{V}_{\text {population }}=\left(\sum_{i, j=1}^{4} r_{i} \vec{D}_{j}\right) / 4 \\
\vec{D}_{j \text { (optimized) }}=\left[\left(\sum_{i=1}^{4} C_{r_{i}}\right) / 4\right]^{-1} \cdot\left(\sum_{i=1}^{4} r_{i j} \cdot \vec{S}_{j}\right) / 4 .
\end{gathered}
$$

where $i=$ neuron number and $j=$ saccade choice. The optimized saccade vector $\left(\vec{D}_{j \text { (optimized) }}\right)$ was determined by multiplying an inverse correlation matrix $\left(\left[\left(\sum_{i=1}^{4} C_{r_{i}}\right) / 4\right]^{-1}\right)$ (supplemental Fig. $3 a$, available at www. jneurosci.org as supplemental material) of the discharge rates for all possible saccade choices by another correlation matrix (supplemental Fig. $3 b$, available at www.jneurosci.org as supplemental material) between four saccade unit vectors $\left(\vec{S}_{j}\right)$ and neuronal responses $\left(r_{i j}\right)$. Once we obtained the optimized saccade vectors, we multiplied the neuronal responses $\left(r_{i}\right)$ by the optimized saccade vectors and averaged them. This calculation resulted in an optimized $\vec{V}_{\text {population }}$ that took into account the inhomogeneity of the saccade choice locations represented by the sample of neuronal discharges. As for the PVA, we considered the OLE as correctly predicting the saccade choice when the angle between the $\vec{V}_{\text {population }}$ and the actual saccade choice was smallest (supplemental Fig. 4, available at www. jneurosci.org as supplemental material).

\section{Results}

Trained monkeys performed a pop-out selection task in which one stimulus from among four was uniquely identified as the target because of its color. In some arrays, the target appeared red and the three distractors appeared green. In other arrays, the target appeared green and the three distractors appeared red. The color of the target varied from experimental day to experimental day. The position of the target within the array of four stimuli appeared at a random location within experimental days. Therefore, for each trial, the monkeys knew the color of the target, but they did not know the position of the target. Figure $1 a$ shows an example of the task in which the target appeared red. We recorded from four neurons simultaneously while monkeys performed this task. Each stimulus position appeared in the empirical center of the RF of the recorded neuron. As a result, the positions of the stimuli in the array were constrained by the positions of the electrodes within the SC map, although two were always located in each SC and we excluded neurons with overlapping RFs (Fig. $1 b$ and see Materials and Methods). Figure $1 a$ shows the idealized case in which the stimuli appeared $90^{\circ}$ from one another. For data presentation, we normalized the positions so that each of four spots appeared at $45^{\circ}, 135,225$, and $315^{\circ}$.

Because of the variability in the positions of the targets, there was variability in choice performance (Kim and Basso, 2008). In the example shown in Figure $1 a$, the monkey performed with $100 \%$ accuracy on trials when the target appeared at location $45^{\circ}$. On the same experimental day, performance was between 70 and $99 \%$ accurate on trials when the target appeared at the $315^{\circ}$ position. Performance was poorer ( $<70 \%$ accurate) when the target appeared at position $225^{\circ}$. Figure $1 c$ shows an example of the recordings from four neurons in this task. We demonstrated recently that the relative level of activity of SC target neurons and SC distractor neurons predicts saccade accuracy in a manner consistent with the interpretation that SC neuronal activity encodes the saccade choice (Kim and Basso, 2008). Furthermore, the range of choice probabilities we and others obtained from SC neurons was similar to the range reported in decision making tasks in SC and other brain regions such as FEF and lateral intraparietal area (Horwitz and Newsome, 2001; Shadlen and Newsome, 2001; Gold and Shadlen, 2002; Horwitz et al., 2004). This suggests that similar numbers of neurons in these areas are pooled to determine the choice.

\section{SC population activity and saccade choice}

Previous work in SC indicates that buildup/prelude neuronal activity scales with the likelihood of saccade occurrence (Basso and Wurtz, 1998; Dorris and Munoz, 1998; Kim and Basso, 2008), suggesting that saccade choice may be encoded across the population of SC buildup neurons. We formulated three models to reveal insights into the computational principles underlying saccade choice in SC. For each model, we compared the results of the model with the saccade choice made by the monkeys to determine the models' accuracy. We implemented a simple WTA model, two variants of the population vector average model (PVA and OLE), and a probabilistic model based on Bayesian inference called the MAP. These models are explained in Materials and Methods. In what follows, we describe the results of each model. In parallel, we point out important extensions of the probabilistic model we implemented compared to that implemented by others.

\section{Probabilistic and WTA models}

One important point about computing the MAP is the manner in which $P(r \mid s)$ (the likelihood) is estimated. We did this in two different ways. First, we used a Poisson PDD to characterize variability for $P(r \mid s)$ as depicted in Figure $2 a-d$ and as done recently by others (Jazayeri and Movshon, 2006; Beck et al., 2008). Second, we determined $P(r \mid s)$ directly using nonparametric density 
estimation (see Materials and Methods and Fig. 2e,f). When estimating $P(r \mid s)$ using a Poisson PDD to characterize variability, we constrained the distribution in three different ways. In one way, we used the log of Gaussian functions that were designed to simulate the tuning curves of SC neurons with parameters $\sigma=$ $20.6^{\circ}$ and baseline discharge $=7$ spikes $/ \mathrm{s}$ (Fig. 2b) (Edelman and Keller, 1998). Note that recent work used von Mises curves for which the logs are cosine functions (Jazayeri and Movshon, 2006; Beck et al., 2008). In a second way, we estimated the tuning curves by measuring the discharge rates of neurons during performance of saccades to the different target positions when only a single target appeared. In the third way, we used the discharge of SC neurons when saccades were made in the four possible target conditions to estimate the tuning curves (Fig. 2d). Importantly, for the tuning curves measured from the four possible target trials, we used the same data for estimating tuning curves and predicting movement choice. Therefore, we implemented a leave-one-out cross-validation procedure to avoid model overestimation (see Materials and Methods).

Figure 2 illustrates these different approaches to estimating (log) likelihoods. In these examples, individual neurons are indicated by different colors, and the condition shown is when the spot located in the $45^{\circ}$ position was the target. For this condition, the red neuron contributes little discharge (Fig. 2a,c, red dot). Likewise, the green and black neurons contribute little since these neurons are largely inactive for this target position. The blue neuron contributes maximally since the target is located within the center of its RF (Fig. 2a,c, blue dot). Note that in Figure 2, $a$ and $c$, we interpolated discharge rate points in between the four target positions to generate smooth functions for the discharge rates. This was done for display only. In reality there are only four points, one for each of the four possible target conditions. These discharge rates are then weighted by the logs of the individual tuning curves, either modeled as Gaussian functions (Fig. 2b), estimated from the real data (Fig. $2 d$ ) or determined directly from the probability density estimation procedure using the real data (Fig. 2e,f). Combining the (log) likelihoods (Fig. 2g) with the prior probability (Fig. $2 h, i$ and see Materials and Methods) yields the posterior probability across all four possible saccade choices (Fig. 2j,k). The same computation excluding the prior yields a log likelihood distribution (Fig. 2g).

All models used simultaneously recorded neuronal data measured in $20 \mathrm{~ms}$ epochs $28 \mathrm{~ms}$ to $8 \mathrm{~ms}$ before the onset of the saccade on a trial-by-trial basis for all 120 neurons ( 30 datasets of 4 neurons) from two monkeys. Figure $3 a$ shows the result of the MAP model with simulated Gaussian tuning curves. The black line shows the mean of the MAP estimates of all the trials for each of the four possible target positions in which the saccade choice was predicted correctly by the maximum of the posterior probability distribution. The gray line shows the same for the trials in which the saccade choice was not predicted by the MAP estimates. Overall the MAP estimates predicted saccade choices on $2870 / 4035(71.11 \%)$ trials. Having the neuronal response of each neuron weighted by the log of a uniform Gaussian (or uniform cosine functions) means that the contribution of each neuron was directly proportional to its discharge rate. Since the Gaussian tuning curves were shifted by $90^{\circ}$, they were minimally overlapping, and therefore the distractor neurons contributed an identical and minimal amount to all conditions. Thus, the neuron with the highest discharge rate dominates the prediction of saccade choice in the posterior distribution. This result is, in principle, identical to the prediction of a WTA model.
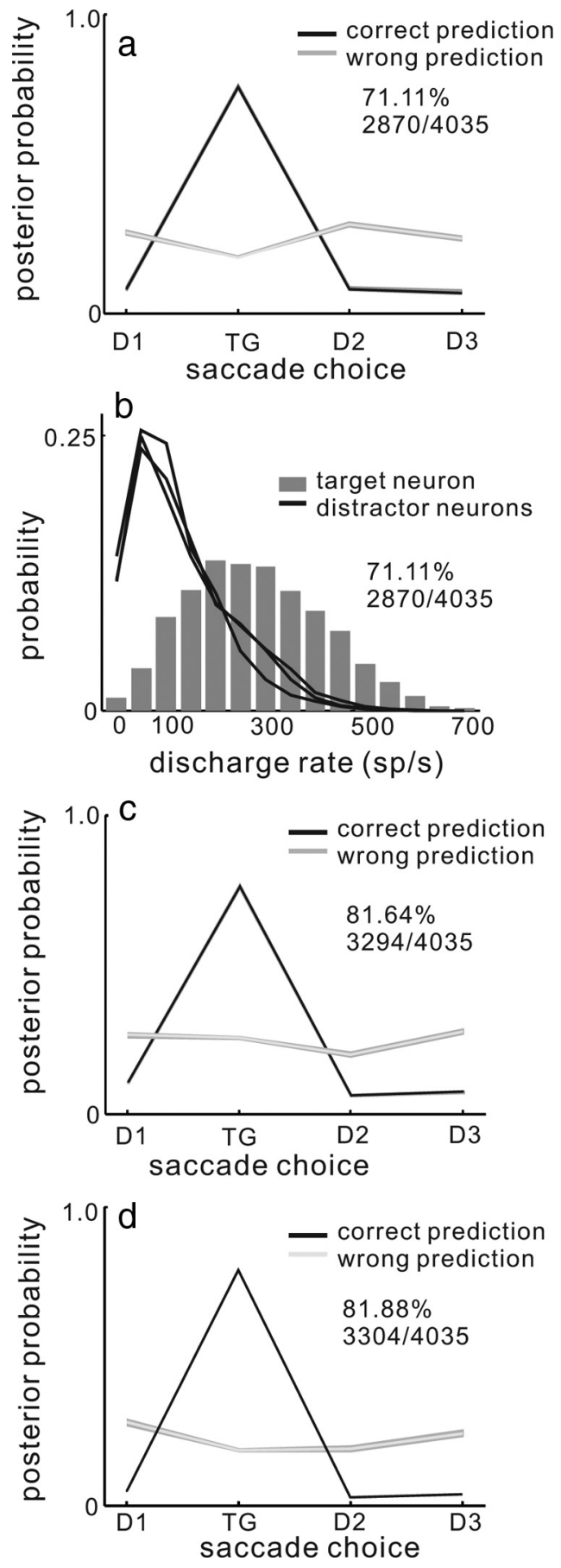

Figure 3. WTA and MAP models of saccade choice. $\boldsymbol{a}$, The mean of the MAP estimates across all trials, computed using the logs of fixed Gaussians as weights is plotted for the four possible saccade choices. The black line shows the mean of the MAP estimates for the trials in which the model estimate correctly predicted the saccade choice. The gray line shows the mean of MAP estimates across all the trials in which the model estimate did not predict saccade choice correctly. The shading around the lines indicates 1 SE. This version of the MAP model could predict $71.11 \%$ ( 2870 of 4035 trials) of all saccade choices. $\boldsymbol{b}$, The probability of measuring a particular discharge rate in the interval 28 to $8 \mathrm{~ms}$ before saccade onset in the selection task is plotted for all neurons across all 30 datasets. Black lines show probability of nonselected neuron discharge rates. The gray bars show probability of selected neuron discharge rates. The WTA model could predict $71.11 \%$ (2870 of 4035 trials) of all saccade choices. $\boldsymbol{c}$, The same as in $\boldsymbol{a}$ except the posterior probability was computed using the log of the tuning curves of $\mathrm{SC}$ neurons to characterize $P(r \mid s)$. Saccade choices were predicted with $81.64 \%$ (3294/4035) accuracy. $\boldsymbol{d}$, Same as in $\boldsymbol{a}$ and $\boldsymbol{c}$ except the posterior was computed using the empirical probability distribution to characterize $P(r \mid s)$. Saccade choices were correctly predicted with $81.88 \%(3304 / 4035)$ accuracy. 
Figure $3 b$ plots frequency histograms of the mean discharge rate in each of the 4035 trials from all 120 neurons. In the WTA model the neuron with the highest discharge rate predicts saccade choice. As is evident from Figure 3b, the distribution of discharge rates measured from target neurons overlaps with the distribution of discharge rates measured from distractor neurons (Fig. 3b, compare black lines and gray bars). This indicates that the distractor neurons were as likely to have the highest discharge rate as the target neurons for some trials. This occurred even though the saccade choice corresponded to the RF location of the target neuron on all of the trials from which these data were taken. Overall $71.11 \%(2870 / 4035)$ of saccade choices were predicted correctly by the WTA model.

The homogeneous and nonoverlapping tuning curves used in this version of the MAP model do not provide any benefit over the WTA model in predicting saccade choice. This result also reveals the possibility that simulated, nonoverlapping and homogeneous tuning curves may not be optimal for population coding. To explore whether using the actual neuronal tuning curves would improve the model predictions over simulated ones, we implemented a version of the MAP model using tuning curves estimated from actual neuronal responses. In Figure $3 c$, we show the result of the MAP model when the tuning curves were estimated from the SC neuronal discharge in the four possible target conditions. In this case, the tuning curves "overlap" in the sense that there is discharge in the distractor neurons because there is always a stimulus in their RF, whether or not that stimulus will ultimately be chosen for a saccade. This implementation of the MAP model improved accuracy by $\sim 10 \%$ over the previous version in that it predicted saccade choice on $3294 / 4035$ (81.64\%) of trials compared to $71.11 \%$ of the trials. This provides some indication that the properties and amount of overlap in the distributions used to estimate the likelihood (logs of tuning curves in this case) is an important variable when considering population coding of movement choice.

Although we used the tuning curves estimated from the neuronal data, the implementation of the MAP model described above still made the assumption that the variability used to estimate $P(r \mid s)$ could be characterized by a Poisson probability distribution. However, the Poisson assumption is unrealistic biologically for single neurons. This is unrealistic, particularly for SC neurons, where discharges are characterized by robust bursts shortly before and during the generation of saccades (see supplemental Fig. 1 $a$, available at www.jneurosci.org as supplemental material) (Sparks, 1986; Moschovakis et al., 1996). In light of this, we implemented an additional version of the MAP in which we determined $P(r \mid s)$ directly from our recorded neuronal data by generating a probability distribution of the neuronal discharges (Fig. 2e,f and see Materials and Methods). Estimating $P(r \mid s)$ directly from the raw discharges improved the result of the MAP model minimally. Overall it predicted saccade choices correctly in $81.88 \%$ (3304/4035) of trials (Fig. $3 d$ ). That there was little difference between the result using this method and the result using a Poisson probability distribution is consistent with recent theoretical work (Ma et al., 2006; Beck et al., 2007) and suggests that even though individual neuronal variability is not well explained by Poisson statistics, a MAP estimate based on Poisson variability performs well.

\section{MAP estimates with the nonuniform priors}

Up to now, we recovered the MAP estimates using a uniform prior and the empirical PDDs to estimate $P(r \mid s)$. We did this because the target for the saccade choice was equally likely to appear in each of the four possible locations on every trial and monkeys did not have to use a before perform this task. However, despite the correct target occurring with a $25 \%$ probability, monkeys did not always perform with this accuracy indicating that they likely incorporate biases into their choices. These biases must be based on something other than the sensory information (Kim and Basso, 2008). Therefore, we implemented a nonuniform prior distribution into our MAP model to determine whether we could improve the MAP estimates of saccade choice. As described in the Materials and Methods, we simulated many possible prior distributions and identified one for each dataset that resulted in the maximum number of correct MAP estimates. To validate our use of these simulated distributions, we compared them to the distributions of saccade choices the monkeys actually made. Note that the prior distributions and the distributions of saccade choices are not identical, but they should be related. Although the actual prior distribution used by monkeys is unknown, we reasoned that correlations between the simulated prior distributions and the distributions of saccade choices would indicate a reasonable approximation to the actual prior. In Figure $4 a$, we show that across the sample, the simulated prior distributions were correlated to the distributions of saccade choices. An example of one such correlation is shown in the inset of Figure $4 a$. Each point in Figure $4 a$ is a pair of points, one from the distribution of saccade choices and one from one of the simulated prior distributions. The arrow drawn from the inset to the point in Figure $4 a$ shows the two points from the inset remapped to the plot of points shown in Figure $4 a$. The total number of points in Figure $4 a$ is 120 because we had 30 datasets and each dataset has four possible saccade choices. One simulated prior distribution was used for each dataset.

Figure $4 b$ shows the result of adding the nonuniform prior distribution to recover the MAP estimate using the empirical PDD to characterize $P(r \mid s)$. The black line shows the mean of the MAP estimates for trials correctly predicted by the MAP model. The gray line shows the mean of the MAP estimates for trials that were incorrectly predicted by the model. Overall the MAP estimates with the nonuniform prior predicted saccade choices on $3420 / 4035(84.76 \%)$ trials. By using the nonuniform prior, the performance of the MAP estimates improved $2.88 \%$, from $81.88 \%$ to $84.76 \%$. The influence of priors on model performance is highly dependent on task demands. Therefore, we next asked whether the change in prediction accuracy occurred for correct and error trials similarly. We reasoned that monkeys might make more errors because they relied more on their priors than on the sensory information to inform their choice. Therefore, we guessed that MAP estimates of saccade choice might improve preferentially for error trials over correct trials when we used the nonuniform prior. Figure $4 c$ plots the percentage of trials correctly predicted by the MAP estimates using the uniform versus the nonuniform priors sorted by whether the trial was correct or in error. For correct trials, the MAP estimates with the uniform prior predicted choice in $84.91 \%$ of the total trials (Fig. $4 c$, second gray bar). Using the nonuniform prior increased the prediction accuracy to $87.22 \%$. This is an improvement in the MAP estimate of $2.31 \%$. For error trials, the MAP estimates determined with the uniform prior accurately predicted saccade choices on $71.49 \%$ of trials (Fig. $4 c$, third gray bar). The MAP estimates determined with the nonuniform prior accurately predicted $76.32 \%$ of the error trials. This represents an improvement in the model accuracy by $4.83 \%$ (Fig. $4 c$, third black bar). We performed a resampling procedure in which we randomly selected correct trials and error trials and assessed whether they 

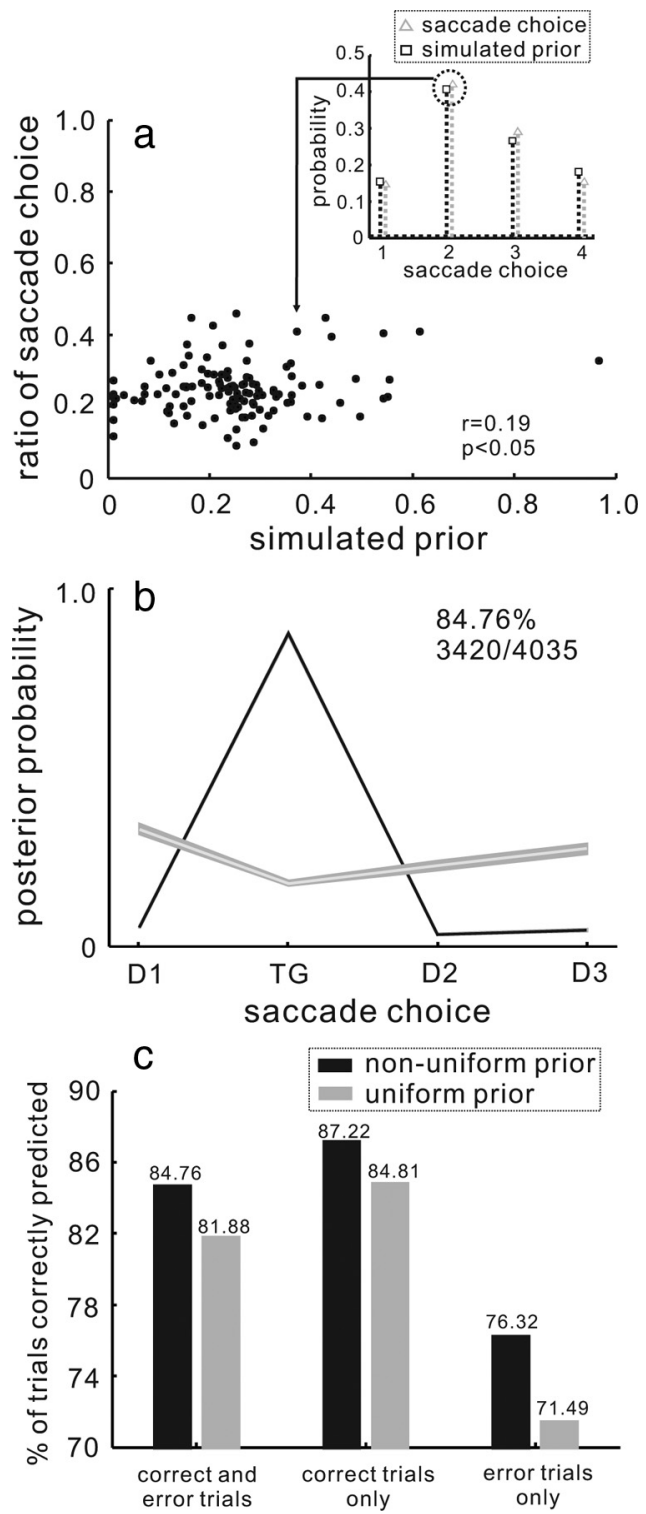

Figure 4. The influence of the prior in predicting a saccade choice. $\boldsymbol{a}$, The ratio of the saccade choice is plotted against the simulated prior probability. One example of choice distribution and the simulated prior distribution appears in the inset (black dotted line and squares = simulated prior; gray dotted line and triangles = the distribution of saccade choices). To show the relationship between the distribution of saccade choices and the simulated prior distribution, two points from the inset were remapped to the plot of points shown in $\boldsymbol{a}$. This was done for all 120 datasets, so $n=120$ points. The Pearson correlation coefficient was determined, $r=0.19$. This relationship was small but statically significant $(p<0.05) \boldsymbol{b}$, The mean of the MAP estimates across all trials, computed using the empirical PDD, and the nonuniform prior distribution is plotted for the four possible saccade choices. The black line shows the mean of the MAP estimates for the trials in which the model estimate correctly predicted the saccade choice. The gray line shows the mean of MAP estimates across all the trials in which the model estimate did not predict saccade choice correctly. Shading around the lines indicates $1 \mathrm{SE}$. This version of MAP model predicted the saccade choice correctly on 3420/4035 (84.76\%) trials. c, The percentage of trials predicted correctly from the MAP model is plotted for the models using the uniform (gray bars) and nonuniform (black bars) prior distribution. The trials are sorted by whether they were correct or in error. The percentages in each condition appear on the top of each bar.

were predicted by the MAP model with the uniform or the nonuniform prior. Repeating this sampling procedure 1000 times and comparing the resulting distributions indicated that the improvement in prediction accuracy that occurred by implementing a nonuniform prior to recover the MAP estimate occurred more for error trials than for correct trials. The differences in

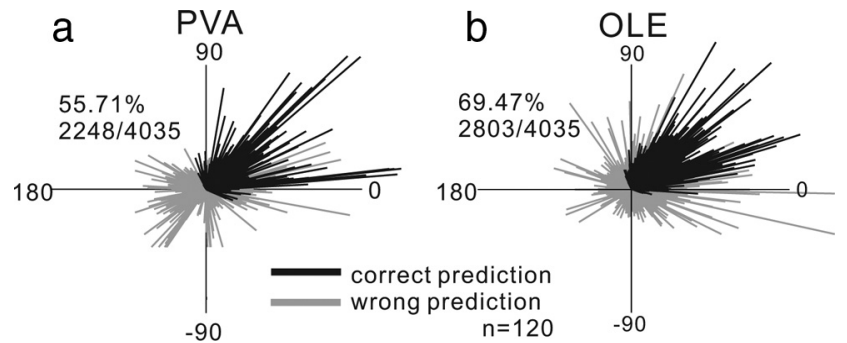

Figure 5. Population vector average and saccade choice. $\boldsymbol{a}$, The neuronal vector determined by Equation 13 . The direction of each line indicates the direction of the population vector and the length indicates the strength of activity. The black lines show the trials in which the population vector correctly predicted saccade choice. The gray lines show the incorrectly predicted choice. PVA correctly predicted $55.71 \%$ (2248/4035) of trials. $\boldsymbol{b}$, Same as in $\boldsymbol{a}$ for the $0 \mathrm{LE}$ computed as described in Equation 14. The OLE correctly predicted $69.47 \%$ (2803/4035) of trials.

improvements were statistically significant $\left(\chi^{2}=1000, p<\right.$ $0.001)$. Based on these results, we conclude that the prediction accuracy of the MAP estimates using a nonuniform prior exceeds that of the uniform prior. Furthermore, using a nonuniform prior improves the MAP estimates preferentially for error trials over correct trials. This suggests that errors in choice may occur because monkeys base their choices on the prior information rather than the sensory information.

\section{PVA and OLE}

In the same way that motor cortex is considered to encode arm movement direction (Georgopoulos et al., 1986; Schwartz et al., 1988), it is considered that the SC encodes the direction of a saccade by averaging across the population of active neurons, each of which contributes a minivector to determine saccade direction. Although supported by lesion experiments (Lee et al., 1988; Quaia et al., 1998; Hanes and Wurtz, 2001) and argued on theoretical grounds (Van Gisbergen et al., 1987; McIlwain, 1991; Groh, 2001), recent lesion experiments (McPeek and Keller, 2002) and dual neuron recording experiments (Port and Wurtz, 2003) suggest that a vector average may be too simplistic, at least when considering saccades made in the presence of more than one visual stimulus. Therefore, we implemented a PVA model as well as an improved version, the OLE, to assess whether these models could predict saccade choice as well as the MAP model.

For the PVA and OLE the same dataset was used as that used for the MAP model. Figure $5 a$ shows the result of the PVA. Figure $5 b$ shows the result of the OLE. Each line is the neuronal population vector for each trial $(n=4035)$. The black lines show the result when the direction of the population vector and the direction of the saccade had the smallest angular difference (see supplemental Fig. 4, available at www.jneurosci.org as supplemental material). We considered these trials to have a correct prediction. The gray lines show the result when the difference in the angle of the direction of the population vector and the angle of the direction of one of the distractor stimuli was the smallest. We considered these trials to have an incorrect prediction. Overall the PVA accurately predicted $2248 / 4035$ (55.71\%) of saccade choices (Fig. 5a).

The PVA predicted the saccade choice correctly for many trials but also failed quite often. Next, we optimized the neuronal vectors with the correlation matrix. This maximizes the PVA performance by taking into account the fact that our sample of neurons did not contain a homogenous representation of saccade space (see Materials and Methods). Figure $5 b$ shows the prediction results of the OLE. Overall the OLE predicted 2803/4035 $(69.47 \%)$ of saccade choices accurately, which represents a 


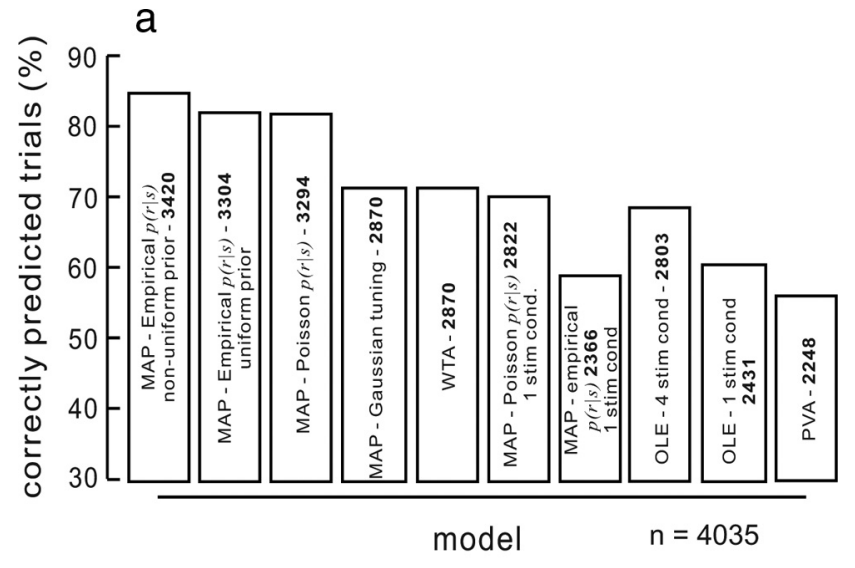

b

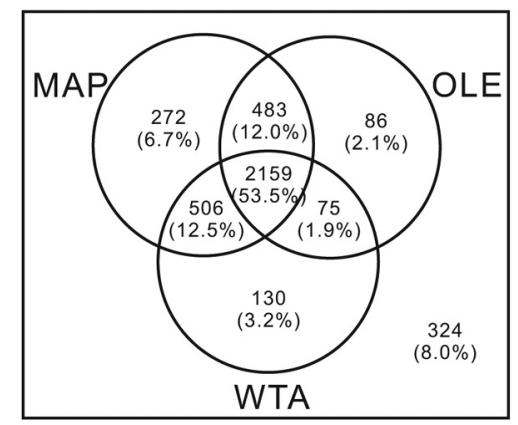

Figure 6. Comparing models of saccade choice. $\boldsymbol{a}$, The percentage of trials predicted correctly from each of the models is plotted. The number inside each bar indicates the number of trials (out of the total 4035) that were correctly predicted by each model. Each model result is labeled inside each bar. From left to right, the bars are as follows: MAP with empirical PDD characterization of $P(r \mid s)$ determined from four stimulus condition data with the nonuniform prior; MAP with Poisson PDD characterization of $P(r \mid s)$ determined from four stimulus condition data with the uniform prior; MAP with Poisson PDD constrained by the tuning curves measured from the four stimulus condition data; MAP with Gaussian tuning curves; WTA; MAP with Poisson PDD characterization of $P(r \mid s)$ determined from single stimulus condition data; MAP using empirical PDD characterization of $P(r \mid s)$ using single stimulus condition data; OLE optimized with four stimulus condition data; OLE optimized with single stimulus condition data; and PVA. $\boldsymbol{b}$, Venn diagram of the percentage of trials predicted by each of the models. The MAP model in this plot is the one using the empirical PDD characterization of $P(r \mid s)$ determined from the four stimulus condition data and the nonuniform prior (the first bar in the top panel). None of the models predicted $8.0 \%$ (324/4035) of the trials.

13.76\% improvement over the PVA. However, when compared to the WTA and MAP models, the OLE showed the lowest prediction accuracy of saccade choice. Note that this is despite the fact that like the MAP, the OLE uses information from all four neurons to determine the saccade choice.

Figure 6 provides a direct comparison of the results from all the models and their different implementations. The MAP using a nonparametric density estimation procedure (from data recorded in the four possible target condition) for determining $P(r \mid s)$ and a nonuniform prior predicts saccade choices very well. This version of MAP predicted saccade choices correctly in $84.76 \%$ (3420/4035) of all the trials (Fig. $6 a$, first bar). The next best prediction occurred for the same implementation with the uniform prior. This version predicted $81.88 \%$ (3304/4035) of all trials (Fig. $6 a$, second bar). When we estimated $P(r \mid s)$ using a Poisson PDD constrained by the tuning curves measured from the four stimulus condition data, the MAP estimate predicted saccade choices equally well at $81.64 \%$ (3294/4035) of all trials (Fig. $6 a$, third bar). The MAP with Gaussian tuning curves and WTA had identical results, both correctly predicting saccade choices in 71.11\% (2870/4035) of all trials (Fig. 6a, fourth and fifth bars). When we used the data from the single target condition for the tuning curves and the Poisson probability density, MAP predicted saccade choices in $69.94 \%(2822 / 4035)$ of the trials. This was a drop in model performance by $14.82 \%$ compared to the best MAP prediction (69.94\% vs $84.76 \% \%$ ) (Fig. $6 a$, first and sixth bars). The model performance degraded further when we determined $P(r \mid s)$ from the nonparametric density function built from the data collected during performance of the single target condition. In this case, 58.64\% (2366/4035) of all trials were predicted from the trials (Fig. $6 a$, seventh bar).

When the OLE was optimized using the data from the four stimuli condition it predicted saccade choice for $69.47 \%$ (2803/ 4035 ) of all trials (Fig. $6 a$, eighth bar). This result is about as good as the WTA $(69.47 \%$ vs $71.11 \%)$. However, when the OLE was optimized using the data recorded during the single target condition, its performance dropped to 60.25\% (2431/4035) (Fig. 6a, ninth bar). As expected, the PVA performed least well, predicting $55.71 \%(2248 / 4035)$ of all trials (Fig. $6 a$, tenth bar). Together these results point toward two important conclusions. First, the model of the population code from the SC build up neurons that can be used to predict choice improves when it combines all the information about the neurons in the population such as their full tuning curves and not just the peak of the tuning curve as is the case for the traditional population vector average. Second, a critical aspect that determines the performance of the model of the population code is the distribution from which $P(r \mid s)$ is drawn. The reason that the accuracy of the models using the single target neuron data performed so poorly is because these data are not accurate characterizations of $P(r \mid s)$ in the four possible target condition.

The Venn diagram in Figure $6 b$ shows the percentages of trials predicted by each model and how the predictions overlapped. Determining whether there are substantial numbers of trials predicted exclusively by one model rather than another provides important information about the computational principles underlying population coding and choice in the SC. We selected three models that showed the best prediction accuracy: the MAP with nonparametric PDD estimation from the four stimuli condition and the nonuniform prior, the OLE optimized with the four stimuli condition and the WTA. Saccade choices were predicted successfully in $92.0 \%$ of all trials by any of the models, whereas $8.0 \%$ of all trials were unpredictable by any of the models. For $53.5 \%$ of all trials, each model did a good job at predicting saccade choice. The MAP predicted $6.7 \%$ of trials exclusively, whereas OLE predicted $2.1 \%$ of trials exclusively, and WTA predicted $3.2 \%$ of trials exclusively. Thus, although all models perform reasonably well, the probabilistic model overall performs slightly better than both of the others. Future experiments with target locations represented by overlapping neuronal RFs will provide further and better tests of the ability of MAP estimates to predict choices relative to these other models.

\section{The posterior probability distribution scales with choice performance accuracy}

Previously we showed that for buildup neurons encoding targets and distractors the levels of activity scaled with performance accuracy (Kim and Basso, 2008). When performance accuracy was high the differences in discharge rates between target and distractor neurons were highly discriminable. When performance accuracy was poor, the differences in discharge rates were less discriminable. In light of this, we were also interested in determining whether the posterior probability from the MAP model 
and the angular difference from the OLE varied with the variability in behavioral performance. Since these two models are based on combining activity from multiple neurons, we expected that the output of these models would scale with performance accuracy. In the case of the OLE, we expected to see a small angular difference between the population vector and the saccade choice when performance accuracy was high. We expected to see a larger angular difference when performance accuracy was poor. For the MAP, we expected to see the peak of the posterior distribution centered on the saccade choice with a higher probability when performance accuracy was high. When performance accuracy was low, we still expected to see the peak of the posterior distribution centered on the saccade choice but with a lower probability. Note that the area under the distribution would remain 1, but the relative probabilities associated with each saccade choice would differ with choice accuracy.

To explore the relationship between the variability of behavioral performance and the model predictions, we sorted all of the correct trials $(n=3317)$ from the 30 datasets into three bins of performance accuracy, $<70 \%$ correct $(n=737), 70-99 \%$ correct ( $n=1255)$, and $100 \%$ correct $(n=1325)$. We then fed the neuronal discharge data measured from 28 to $8 \mathrm{~ms}$ before the onset of the saccade from these sorted trials into both the MAP and the OLE models. Figure $7 a$ shows the angular difference in the directions of the population vector and the four possible saccade choices. Because we sorted these trials from only correct trials for this analysis, the target direction shows a much smaller angular difference than any of the other locations. However, when we compared the angular difference for the target location across the different performance conditions, there were small changes in the difference, but they failed to reach statistical significance (ANOVA; $F_{(2,117)}=1.02, p=0.363$ ) (Fig. $7 a$, compare black and gray lines). In contrast to the OLE, the posterior distribution showed scaling with performance accuracy, and these differences were statistically significant (Fig. $7 b$, compare black and gray lines) (ANOVA $F_{(2,117)}=3.98, p<0.05$ ). Previously, we found that the activity of SC neurons encodes saccade choice as well as the certainty of the choice (Basso and Wurtz, 1997, 1998; Kim and Basso, 2008). The findings described here corroborate and extend that result showing that the posterior distribution recovered from combining likelihoods obtained from SC neuronal activity and prior information predicts saccade choices and scales with choice accuracy. Inherent in the posterior distribution is the certainty of the choice indicated by the performance accuracy.

In one implementation of the MAP model, we used a uniform prior probability distribution. Because of this, the result of this model is mathematically equivalent to that of likelihood models (Sanger, 2002, 2003) most recently implemented in MT (Jazayeri and Movshon, 2006). Figure $7 c$ plots the log likelihood distributions for each of the three performance conditions so that we could compare directly the MAP to the log likelihood. Although not intuitive, the distributions shown in Figure $7 c$ reveal a pattern. When performance accuracy was low the distribution did not have a clear peak (Fig. $7 c$, lightest gray line). Whereas when performance accuracy was high, the log likelihood distribution was centered on the saccade choice (Fig. $7 c$, black line). However, because these are likelihoods and not bona fide probability distributions, they cannot be compared directly across conditions unless they are normalized. Note that the light gray line is for the poorer performance trials even though it has the highest overall log likelihood. A difference between the maximum and minimum log likelihood can be taken for this purpose. Using a pos-
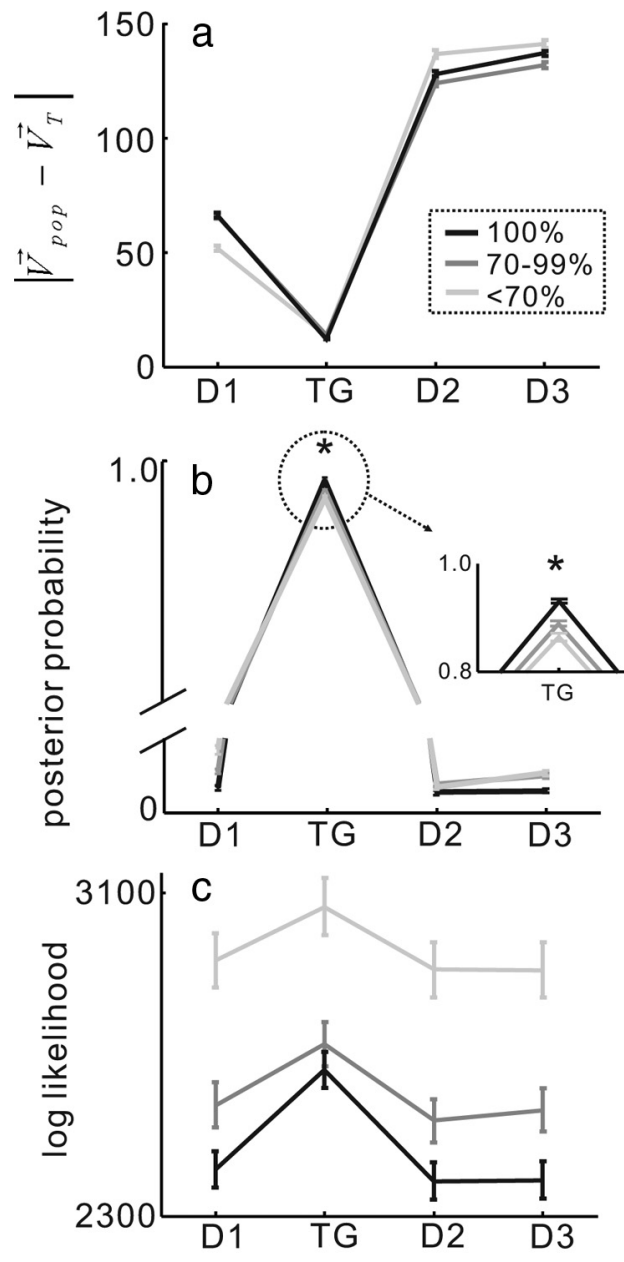

Figure 7. MAP estimates scale with performance accuracy. The black lines show mean angular difference for the trials in which performance was 100\%. The dark gray lines show the mean angular difference for trials in which performance was 70-99\% accurate. The light gray lines show the mean angular difference for trials in which performance accuracy was $<70 \%$. D1 = distractor 1; D2 = distractor 2; D3 = distractor 3; TG = saccade target choice. $\boldsymbol{a}$, The angular difference between the direction of the prediction resulting from the OLE calculation and the actual saccade direction for trials in which performance accuracy varied. $\boldsymbol{b}$, The mean of the MAP estimate with nonuniform prior for the four possible saccade choices, D1, TG, D2 and D3 as in $\boldsymbol{a}$ is plotted for each of the three accuracy conditions. The inset shows the region of the distribution in the dashed circle. ${ }^{*} p<0.05$. c, Log likelihood for each of the four possible saccade choices. The arrangement is the same as in $\boldsymbol{a}$ and $\boldsymbol{b}$.

terior distribution however, which is a bona fide probability distribution, this difference step is not required.

\section{The posterior probability distribution develops over time}

Given that decisions and saccade choices likely develop over time (Carpenter and Williams, 1995; Gold and Shadlen, 2000, 2007), we extended our analysis to determine whether the information encoded in the posterior probability and the OLE developed over time to reflect a single saccade choice. For this, we aligned the trials on the time beginning $50 \mathrm{~ms}$ (average SC visual latency) after the onset of the stimulus array and computed the model result for each $1 \mathrm{~ms}$ until the saccade onset. Figure $8 a$ plots the angular difference between the neuronal and saccade direction measured using the OLE. Initially, the angular difference fluctuated and began settling on a small difference value $\sim 100 \mathrm{~ms}$ after the onset of the stimulus array. Consistent with the results of the stationary analysis shown in Figure $7 a$, the dynamic OLE predic- 

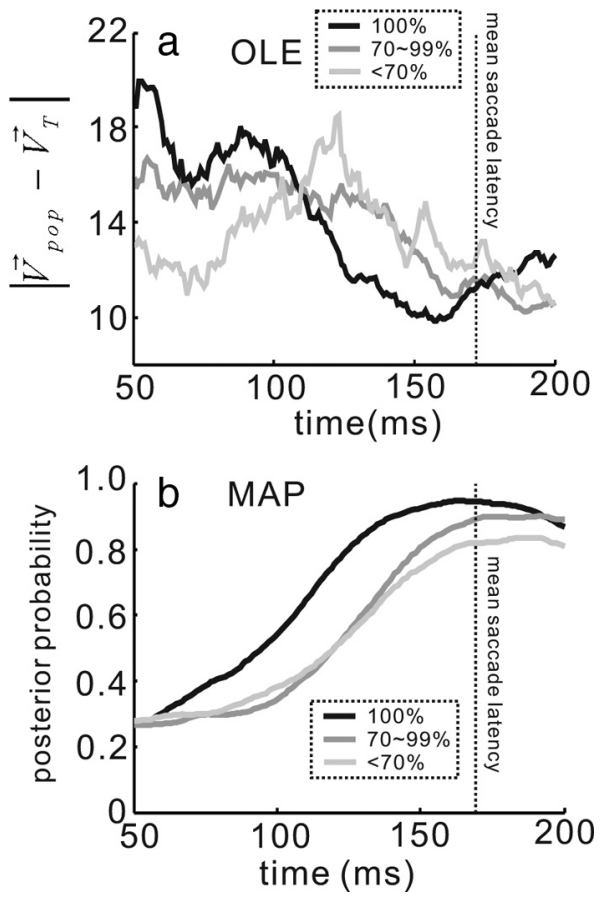

Figure 8. OLE and MAP predictions develop over time. $\boldsymbol{a}$, The angular difference in the direction of the OLE vector and the saccade vector is plotted against time for the three choice accuracy levels. The traces are aligned to the time point $50 \mathrm{~ms}$ after the onset of the stimulus array. Black lines are from the $100 \%$ performance accuracy trials, dark gray lines are the $70-$ $90 \%$ performance accuracy trials, and the light gray lines are the $<70 \%$ performance accuracy trials. $\boldsymbol{b}$, The mean of the MAP estimates for the saccade choice in the three levels of performance accuracy are plotted over time as in $\boldsymbol{a}$. The vertical dotted line in both panels is the mean saccade reaction time $(\mathrm{RT})$.

tion did not scale very well with performance accuracy. In contrast, the developing choice as encoded by the posterior was more obvious in the MAP estimate. Figure $8 b$ shows the developing MAP estimate of the saccade choice in the three performance accuracy conditions ( $<70 \%, 70-99 \%$, and 100\%). The MAP estimate developed rapidly in the $100 \%$ performance accuracy condition and reached a maximum probability of 0.95 at the mean time of the saccade onset (Fig. $8 b$, black line). In the $70-90 \%$ and the $<70 \%$ performance accuracy conditions, the MAP estimate rose less rapidly and reached a probability of 0.90 and 0.83 , respectively, at the mean time of saccade onset (Fig. $8 b$, dark gray and gray lines). The differences in the peak probability at the time of the mean saccade onset were statistically significant (ANOVA, $\left.F_{(2,117)}=5.15, p<0.01\right)$. This result is interesting because it reveals that across the different performance accuracy conditions, the height of the posterior distribution is lower for the poorer performance trials (Fig. $8 b$, light gray line) than for the better performance trials (Fig. $8 b$, black line). In each case, monkeys made the correct saccade choice. This is evidence that the decision or choice threshold varies for these different performance accuracy trials (Hanes and Schall, 1996; Paré and Hanes, 2003).

Figure 9 shows the evolution of the posterior for the target location (TG) and for the three distractor locations (D1, D2, and D3) using both the uniform prior (Fig. $9 a, c, e$ ) and the nonuniform prior (Fig. 9b,d,f). In each panel, the MAP estimate for the saccade choice diverged from the MAP estimates for the distractors locations as saccade onset approached (Fig. 9, compare thick, thin, dashed, and dotted lines). As previously reported in LIP (Beck et al., 2008), these results show the development of the posterior probability of the saccade choice and the decrease of the posterior probabilities of the distractors over time. The results show for the first time that when the nonuniform prior distribution is applied to recover the MAP estimate, the posterior distribution favors the saccade choice slightly, even before the stimulus array appears (Fig. 9, compare $a, c$, and $e$ with $b, d$, and $f$ ). Finally, as would be predicted from a structure that signals the saccade choice to be made, the posterior distribution almost collapses around the saccade choice at the time of the saccade. This is evident from the MAP estimates because the saccade choice probability is close to 1.0 and the probability for the distractors is close to 0 . This behavior is unclear from the dynamic analysis of the OLE shown in Figure $8 a$.

\section{Discussion}

In this report, we show for the first time that the relationship between SC buildup neuronal population activity and saccade choice is well described by a probabilistic scheme. Here, we considered SC buildup neurons as encoders of likelihood distributions. When the likelihoods were combined with prior information, we could construct a posterior distribution over four possible saccade choices whose maximum predicted saccade choices well. Somewhat astonishing to us was that combining the activity of only four simultaneously recorded neurons and recovering the maximum value of the posterior distribution predicted saccade choices accurately on as many as $84.76 \%$ of the trials. The MAP outperformed two well known algorithms, PVA/OLE and WTA. For the first time, we also showed that the posterior distribution across saccade choices develops over time and reached a maximum around the saccade choice at the time of the saccade onset. The posterior distribution almost collapses around the saccade choice at the time of the movement, as would be predicted for a structure so close to the motoneurons (Miyashita and Hikosaka, 1996). We also showed that computing the posterior distribution across saccade choices by estimating the likelihood directly from the data using a probability density estimation procedure maximizes performance of the model. However, consistent with theoretical predictions, the MAP estimates recovered using the empirical PDD were little improved over those using a Poisson distribution to characterize variability. Finally, by incorporating a nonuniform prior, we found that MAP estimates improved by $\sim 3 \%$ across all trials. This suggests that incorporating prior information with the likelihood information provided by SC buildup neurons is a way population neuronal activity could encode saccade choices. In what follows, we first describe how these new results extend previous work on the SC and saccade choice. Then we describe how our MAP model complements and extends previous work on probabilistic approaches to sensory encoding and decision making.

\section{Relationship to previous work in SC}

It is well accepted that the SC employs a population code to determine saccadic eye movements (McIlwain, 1986, 1991). Experimental evidence shows that individual neurons encode saccade vectors, with each neuron having broad tuning for particular saccade directions and amplitudes (Robinson, 1972; Schiller and Stryker, 1972; Wurtz and Goldberg, 1972; Sparks, $1975,1978)$. A weighted sum of the activity of SC neurons across the map is considered to determine the saccade direction (Ottes et al., 1986; Van Gisbergen et al., 1987; Lee et al., 1988; Quaia et al., 1998; Groh, 2001; Hanes and Wurtz, 2001) in much the same way that motor cortical neurons encode the direction of arm movements (Georgopoulos et al., 1986). However, the experi- 
ments leading to this conclusion for the SC are based largely on simulations or were performed using only a single saccade target (Lee et al., 1988; Quaia et al., 1998; Hanes and Wurtz, 2001).

Recent experiments in the saccadic system using more complex displays, such as when multiple visual stimuli appear, suggest that a WTA strategy is used (Port and Wurtz, 2003; McPeek and Keller, 2004). Thus, we are left with the conclusion that for single targets, the SC operates using PVA, whereas for multiple stimuli, the SC operates as WTA. This conundrum is evident from behavioral studies too. For example, it is well known that when two visual stimuli appear in close proximity, saccades land in a location between the two stimuli-a phenomenon called the global effect or averaging saccades (Findlay, 1982; Glimcher and Sparks, 1993; Kowler and Blaser, 1995; Edelman and Keller, 1998; McGowan et al., 1998; Melcher and Kowler, 1999). However, if the targets appear further apart or more time is provided, a saccade can be made to one or the other stimulus (Ottes et al., 1984). This phenomenon is not unique to the SC. In the MT, electrical stimulation and recording experiments support PVA or WTA or both, leading to the idea that perceptual decisions rely on a WTA scheme, whereas movement decisions rely on a PVA scheme (Salzman et al., 1990, 1992; Ferrera and Lisberger, 1997; Groh et al., 1997; Recanzone et al., 1997; Britten and Heuer, 1999; Churchland and Lisberger, 2001). Population coding schemes that are task or time dependent require mechanisms to switch between them. How this switch would be implemented biologically is unclear.

\section{Advantages of probabilistic schemes for understanding action choice}

Probabilistic strategies offer a solution to this conundrum. Because the posterior distribution uses all of the information contained within the tuning curve and it combines activity across all tuning curves, it naturally represents multiple stimuli simultaneously. Furthermore, because the peak and the variance of tuning curves (when considered as likelihood distributions-or the estimated PDDs) signal the certainty of the encoded parameter, the posterior distribution provides a normalized likelihood (conditional probability) for each of the alternatives. This eliminates the need for a switch between population coding schemes. Both PVA and WTA use only the peak activity. WTA further disregards information from distractor neurons, leaving much of the information in the population activity unused. Even when more information is provided for the PVA as in the case of the OLE, it still predicts fewer saccade choices as accurately as the MAP. This is because the OLE does not incorporate variability as does the MAP model. The OLE however, was almost as good as the MAP with the Gaussian tuning curves, which in turn, was uniform prior
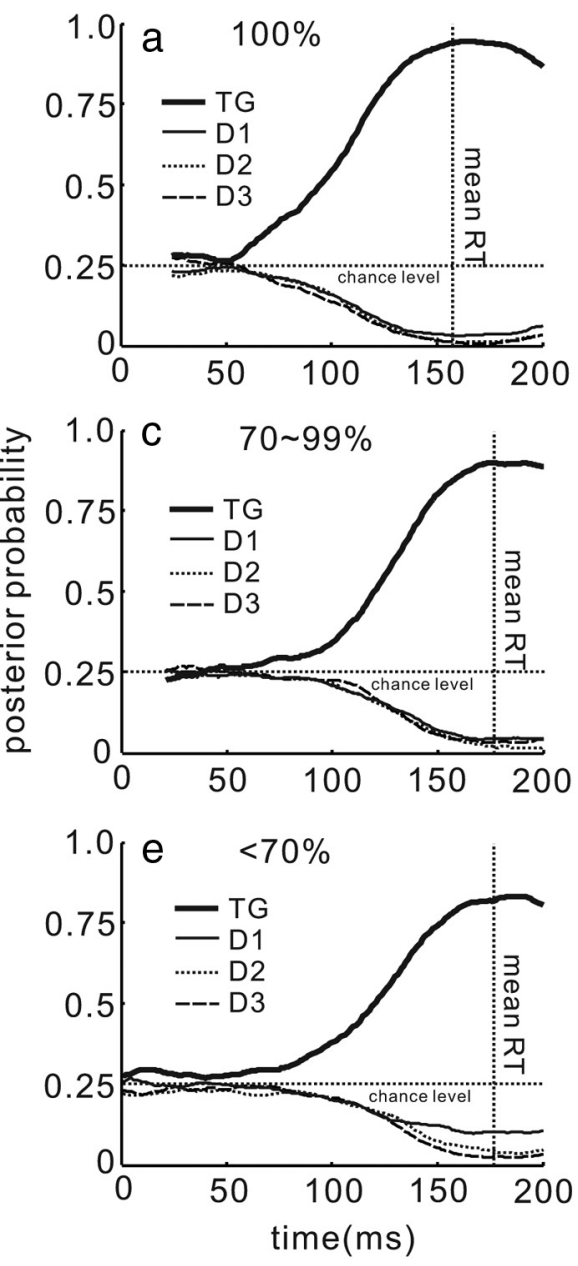

Figure 9. Evolution of the posterior distribution. $\boldsymbol{a}$, The posterior probabilities for the saccade choice (TG) and the three distractor locations (D1, D2, and D3) are plotted over time in the $100 \%$ performance accuracy condition and the uniform prior. , The same as in $\boldsymbol{a}$ for the nonuniform prior. $\boldsymbol{c}$, The same as in $\boldsymbol{a}$ for the $70-99 \%$ performance accuracy condition. $\boldsymbol{d}$, The indicates the mean saccade reaction time (RT).
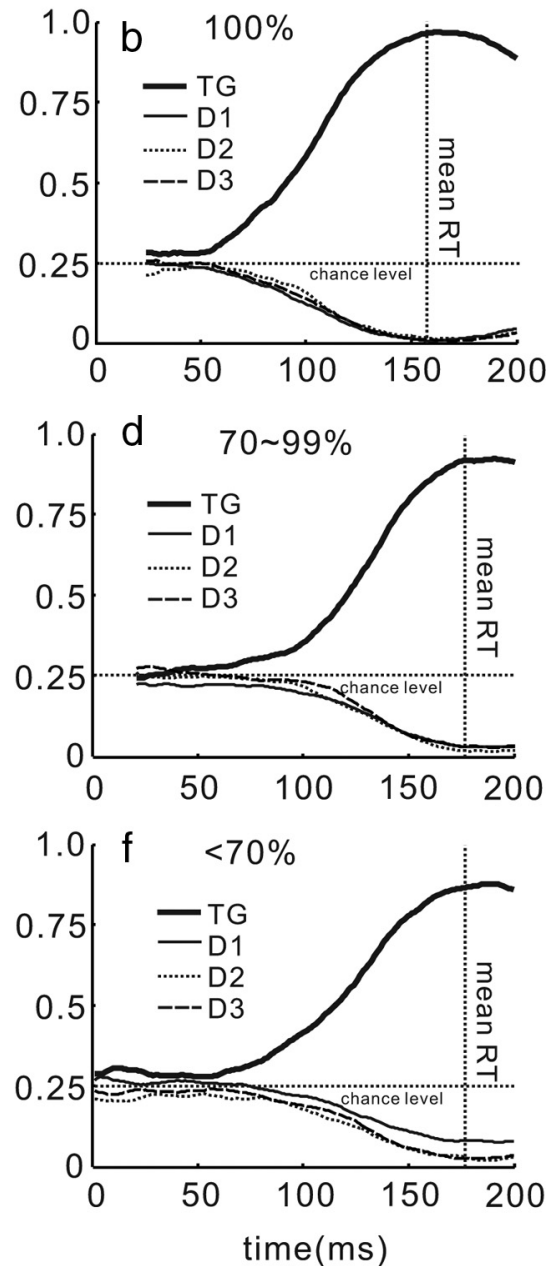

identical to the WTA. This results because the correlation matrix that we used to optimize the vector estimation was determined from neuronal activities that were largely nonoverlapping. Only the empirical PDDs appear to represent the true population variability. Thus we see improvement from the OLE $(69.47 \%)$ and WTA (71.11\%) to the MAP with the empirical PDD $(81.88 \%)$. As a result of ignoring much of the information in the population, variations in behavior, uncertainty, or even attentional modulation (Spitzer et al., 1988; McAdams and Maunsell, 1999; Pouget et al., 1999) cannot be resolved using WTA or PVA/OLE approaches. Future experiments pushing the amount of overlap in the visual display, the RF of the recorded neurons and thus the empirical PDDs, will further distinguish these models.

Probabilistic approaches have the additional advantage in that they are easily extended to the domain of decision making (Smith and Ratcliff, 2004; Gold and Shadlen, 2007; Beck et al., 2008). Current models of decision making or eye movement selection rely on taking the difference of activity from two populations of 
neurons representing independent alternatives (Shadlen and Newsome, 2001; Roitman and Shadlen, 2002; Ratcliff et al., 2003; Huk and Shadlen, 2005; Boucher et al., 2007; Ratcliff et al., 2007). Extending these models to more than two choices or to continuous decisions as opposed to discrete choices, is difficult (Ratcliff et al., 2007; Beck et al., 2008; Churchland et al., 2008; Niwa and Ditterich, 2008). Furthermore, these models have difficulty incorporating changing evidence "on the fly." A neuronal population representing the posterior distribution across choices has the unique advantage of naturally representing multiple possibilities, discrete or continuous variables, the ability for recursive computation (Fig. 8) (Montagnini et al., 2007), and incorporation of prior evidence. These features make changing a mind "on the fly" seamless.

We think of the posterior distribution as describing the probability of a saccade choice. We interpret our result as providing evidence that the saccade choice may be encoded as the posterior probability distribution across all possible saccade choices or the uncertainty associated with each of the possible saccade options. Whereas conceptual models of the decision process suggest that uncertainty underlying decisions occurs primarily within the sensory system (Sugrue et al., 2005), our results suggest that there is uncertainty associated with the choice of action that may be distinguished from sensory uncertainty.

\section{Relationship to previous and current models}

Here we tested some of the theoretical assumptions of similar probabilistic models. We made a number of important findings. First, assuming a Poisson probability distribution to characterize variability to estimate likelihood is as good as determining the likelihood directly from empirical probability density estimation. Therefore, even though individual neuronal variability is not well explained by Poisson statistics, a MAP estimate based on Poisson statistics performs well (Ma et al., 2006; Beck et al., 2007). Second, von Mises or Gaussian tuning may not be the best representation of neuronal activity across the population. We show here that von Mises tuning curves (logs of von Mises curves are cosine functions) are not required for the MAP to perform well. Similarly, using Gaussian tuning curves resulted in model performance that was only as good as WTA. Estimating the likelihoods empirically improved model performance. This result is consistent with results seen in the arm movement literature (Amirikian and Georgopoulos, 2000; Serruya et al., 2002; Taylor et al., 2002; Carmena et al., 2003). That von Mises tuning curves and the minimally overlapping Gaussian tuning curves result in performance identical to WTA is expected since these approaches minimize or negatively weight (in the case of cosine functions) the activity of distractor neurons. Indeed, in recent work extending a Bayesian approach to decision making (Beck et al., 2008), they assumed von Mises curves (the logs of which are cosine functions) for the LIP neurons. We suspect they would obtain the same results as they did if they also implemented a WTA scheme.

Third, we implemented both a uniform and a nonuniform prior probability distribution. Therefore, the MAP estimate in the former case is a Bayesian estimator that is identical to a maximum likelihood estimator and the MAP estimate in the latter case is a Bayesian estimator distinct from a maximum likelihood. Although the addition of the prior did not improve the model enormously, we suspect this is because in our task it was not to the monkeys' advantage to use a prior. Consistent with this, we found more improvement for error trials than for correct trials when the nonuniform prior was added.
In net, we hypothesize that SC buildup neurons encode likelihood distributions as demonstrated here. These in turn may be integrated by burst neurons in SC together with priors (or biases) from other sources to determine the posterior probability distribution across saccade choices. We propose that using bona fide PDDs (such as the empirical PDD) for characterizing $P(r \mid s)$ and encoding saccade choice as a posterior distribution across saccade choices, is a simple way for the brain to represent and compute choices within the population (Deneve et al., 1999). Since the peak of the posterior would be associated with the highest chance of activating the downstream neurons responsible for driving a particular saccade, decoding the choice is implicit in the population activity. Furthermore, the width of the posterior distribution is an implicit way to represent the uncertainty of the choice.

\section{References}

Amirikian B, Georgopoulos AP (2000) Directional tuning profiles of motor cortical cells. Neurosci Res 36:73-79.

Averbeck BB, Latham PE, Pouget A (2006) Neural correlations, population coding and computation. Nat Rev Neurosci 7:358-366.

Basso MA, Wurtz RH (1997) Modulation of neuronal activity by target uncertainty. Nature 389:66-69.

Basso MA, Wurtz RH (1998) Modulation of neuronal activity in superior colliculus by changes in target probability. J Neurosci 18:7519-7534.

Beck JM, Ma WJ, Kiani R, Hanks T, Churchland AK, Roitman J, Shadlen MN, Latham PE, Pouget A (2008) Probabilistic population codes for Bayesian decision making. Neuron 60:1142-1152.

Beck J, Ma WJ, Latham PE, Pouget A (2007) Probabilistic population codes and the exponential family of distributions. Prog Brain Res 165:509-519.

Boucher L, Palmeri TJ, Logan GD, Schall JD (2007) Inhibitory control in mind and brain: an interactive race model of countermanding saccades. Psychol Rev 114:376-397.

Britten KH, Heuer HW (1999) Spatial summation in receptive fields of MT neurons. J Neurosci 19:5074-5084.

Carmena JM, Lebedev MA, Crist RE, O’Doherty JE, Santucci DM, Dimitrov DF, Patil PG, Henriquez CS, Nicolelis MAL (2003) Learning to control a brain-machine interface for reaching and grasping by primates. PLoS Biology 1:e42.

Carpenter RH, Williams ML (1995) Neural computation of log likelihood in control of saccadic eye movements. Nature 377:59-62.

Churchland AK, Kiani R, Shadlen MN (2008) Decision-making with multiple alternatives. Nat Neurosci 11:693-702.

Churchland MM, Lisberger SG (2001) Shifts in the population response in the middle temporal visual area parallel perceptual and motor illusions produced by apparent motion. J Neurosci 21:9387-9402.

Crist CF, Yamasaki DSG, Komatsu H, Wurtz RH (1988) A grid system and a microsyringe for single cell recording. J Neurosci Methods 26:117-122.

Deneve S, Latham PE, Pouget A (1999) Reading population codes: a neural implementation of ideal observers. Nat Neurosci 2:740-745.

Dorris MC, Munoz DP (1998) Saccadic probability influences motor preparation signals and time to saccadic initiation. J Neurosci 18:7015-7026.

Edelman JA, Keller EL (1998) Dependence on target configuration of express saccade-related activity in the primate superior colliculus. J Neurophysiol 80:1407-1426.

Feldman JA (1982) Dynamic connections in neural networks. Biol Cybern 46:27-39.

Ferrera VP, Lisberger SG (1997) Neuronal responses in visual areas MT and MST during smooth pursuit target selection. J Neurophysiol 78:1433-1446.

Findlay JM (1982) Global visual processing for saccadic eye movements. Vision Res 22:1033-1045.

Földiak P (1993) The "ideal homonculus": statistical inference from neural population responses. In: Computation and neural systems (Eeckman F, Bower J, eds), pp 55-60. Norwell, MA: Kluwer Academic.

Fuchs AF, Robinson DA (1966) A method for measuring horizontal and vertical eye movement chronically in the monkey. J Appl Physiol 21:1068-1070.

Georgopoulos AP, Schwartz AB, Kettner RE (1986) Neuronal population coding of movement direction. Science 233:1416-1419. 
Glimcher PW, Sparks DL (1993) Representation of averaging saccades in the superior colliculus of the monkey. Exp Brain Res 95:429-435.

Gold JI, Shadlen MN (2000) Representation of a perceptual decision in developing oculomotor commands. Nature 404:390-394.

Gold JI, Shadlen MN (2002) Banburismus and the brain: decoding the relationship between sensory stimuli, decisions, and reward. Neuron 36:299-308.

Gold JI, Shadlen MN (2007) The neural basis of decision making. Annu Rev Neurosci 30:535-574.

Groh JM (2001) Converting neural signals from place codes to rate codes. Biol Cybern 85:159-165.

Groh JM, Born RT, Newsome WT (1997) How is a sensory map read out? Effects of microstimulation in visual area MT on saccades and smooth pursuit eye movements. J Neurosci 17:4312-4330.

Hanes DP, Schall JD (1996) Neural control of voluntary movement initiation. Science 274:427-430.

Hanes DP, Wurtz RH (2001) Interaction of the frontal eye field and superior colliculus for saccade generation. J Neurophysiol 85:804-815.

Hays AV, Richmond BJ, Optican LM (1982) A UNIX-based multiple process system for real-time data acquisition and control. WESCON Conf Proc 2:1-10.

Horwitz GD, Newsome WT (2001) Target selection for saccadic eye movements: prelude activity in the superior colliculus during a directiondiscrimination task. J Neurophysiol 86:2543-2558.

Horwitz GD, Batista AP, Newsome WT (2004) Representation of an abstract perceptual decision in macaque superior colliculus. J Neurophysiol 91:2281-2296.

Huk AC, Shadlen MN (2005) Neural activity in macaque parietal cortex reflects temporal integration of visual motion signals during perceptual decision making. J Neurosci 25:10420-10436.

Jazayeri M, Movshon JA (2006) Optimal representation of sensory information by neural populations. Nat Neurosci 9:690-696.

Judge SJ, Richmond BJ, Chu FC (1980) Implantation of magnetic search coils for measurement of eye position: an improved method. Vision Res 20:535-538.

Kim B, Basso MA (2008) Saccade target selection in the superior colliculus: a signal detection theory approach. J Neurosci 28:2991-3007.

Kowler E, Blaser E (1995) The accuracy and precision of saccades to small and large targets. Vision Res 35:1741-1754.

Lee C, Rohrer WH, Sparks DL (1988) Population coding of saccadic eye movements by neurons in the superior colliculus. Nature 332:357-360.

Li X, Basso MA (2005) Competitive stimulus interactions within single response fields of superior colliculus neurons. J Neurosci 25:1135711373.

Li X, Basso MA (2008) Preparing to move increases the sensitivity of superior colliculus neurons. J Neurosci 28:4561-4577.

MacPherson JM, Aldridge JW (1979) A quantitative method of computer analysis of spike train data collected from behaving animals. Brain Res 175:183-187.

Ma WJ, Beck JM, Latham PE, Pouget A (2006) Bayesian inference with probabilistic population codes. Nat Neurosci 9:1432-1438.

McAdams CJ, Maunsell JHR (1999) Effects of attention on orientationtuning functions of single neurons in macaque cortical area V4. J Neurosci 19:431-441.

McGowan JW, Kowler E, Sharma A, Chubb C (1998) Saccadic localization of random dot targets. Vision Res 38:895-909.

McIlwain JT (1986) Point images in the visual system: new interest in an old idea. Trends Neurosci 9:354-358.

McIlwain JT (1991) Distributed spatial coding in the superior colliculus: a review. Vis Neurosci 6:3-13.

McPeek RM, Keller EL (2002) Saccade target selection in the superior colliculus during a visual search task. J Neurophysiol 88:2019-2034.

McPeek RM, Keller EL (2004) Deficits in saccade target selection after inactivation of superior colliculus. Nat Neurosci 7:757-763.

Melcher D, Kowler E (1999) Shapes, surfaces and saccades. Vision Res 39:2929-2946.

Miyashita N, Hikosaka O (1996) Minimal synaptic delay in the saccadic output pathway of the superior colliculus studied in awake monkey. Exp Brain Res 112:187-196.

Montagnini A, Mamassian P, Perrinet L, Castet E, Masson GS (2007) Bayesian modeling of dynamic motion integration. J Physiol Paris 101:64-77.
Moschovakis AK, Scudder CA, Highstein SM (1996) The microscopic anatomy and physiology of the mammalian saccadic system. Prog Neurobiol 50:133-254.

Munoz DP, Wurtz RH (1995) Saccade-related activity in monkey superior colliculus. I. Characteristics of burst and buildup cells. J Neurophysiol 73:2313-2333.

Niwa M, Ditterich J (2008) Perceptual decisions between multiple directions of visual motion. J Neurosci 28:4435-4445.

Optican LM, Richmond BJ (1987) Temporal encoding of two-dimensional patterns by single units in primate inferior temporal cortex. III. Information theoretic analysis. J Neurophysiol 57:162-178.

Oram MW, Földiák P, Perrett DI, Sengpiel F (1998) The ideal homunculus': decoding neural population signals. Trends Neurosci 21:259-265.

Ottes FP, Van Gisbergen JAM, Eggermont JJ (1984) Metrics of saccade responses to visual double stimuli: two different modes. Vision Res 24:1169-1179.

Ottes FP, Van Gisbergen JAM, Eggermont JJ (1986) Visuomotor fields of the superior colliculus: a quantitative model. Vision Res 26:857-873.

Paré M, Hanes DP (2003) Controlled movement processing: superior colliculus activity associated with countermanded saccades. J Neurosci 23:6480-6489.

Port NL, Wurtz RH (2003) Sequential activity of simultaneously recorded neurons in the superior colliculus during curved saccades. J Neurophysiol 90:1887-1903.

Pouget A, Deneve S, Ducom J-C, Latham PE (1999) Narrow vs wide tuning curves: what's best for a population code? Neural Comput 11:85-90.

Quaia C, Aizawa H, Optican LM, Wurtz RH (1998) Reversible inactivation of monkey superior colliculus: II. Maps of saccadic deficits. J Neurophysiol 79:2097-2110.

Ratcliff R, Cherian A, Segraves M (2003) A comparison of macaque behavior and superior colliculus neuronal activity to predictions from models of two-choice decisions. J Neurophysiol 90:1392-1407.

Ratcliff R, Hasegawa YT, Hasegawa RP, Smith PL, Segraves MA (2007) Dual diffusion model for single-cell recording data from the superior colliculus in a brightness-discrimination task. J Neurophysiol 97:1756-1774.

Recanzone GH, Wurtz RH, Schwarz U (1997) Responses of MT and MST neurons to one and two moving objects in the receptive field. J Neurophysiol 78:2904-2915.

Robinson DA (1972) Eye movements evoked by collicular stimulation in the alert monkey. Vision Res 12:1795-1808.

Roitman JD, Shadlen MN (2002) Response of neurons in the lateral intraparietal area during a combined visual discrimination reaction time task. J Neurosci 22:9475-9489.

Salinas E, Abbott LF (1994) Vector reconstruction from firing rates. J Comput Neurosci 1:89-107.

Salzman CD, Britten KH, Newsome WT (1990) Cortical microstimulation influences perceptual judgements of motion detection. Nature 346:174-177.

Salzman CD, Murasugi CM, Britten KH, Newsome WT (1992) Microstimulation in visual area MT: effects on direction discrimination performance. J Neurosci 12:2331-2355.

Sanger TD (1996) Probability density estimation for the interpretation of neural population codes. J Neurophysiol 76:2790-2793.

Sanger TD (2002) Decoding neural spike trains: calculating the probability that a spike train and an external signal are related. J Neurophysiol $87: 1659-1663$.

Sanger TD (2003) Neural population codes. Curr Opin Neurobiol 13:238-249.

Scherberger H, Andersen RA (2007) Target selection signals for arm reaching in the posterior parietal cortex. J Neurosci 27:2001-2012.

Schiller PH, Koerner F (1971) Discharge characteristics of single units in superior colliculus of the alert rhesus monkey. J Neurophysiol 34:920-936.

Schiller PH, Stryker M (1972) Single-unit recording and stimulation in superior colliculus of the alert rhesus monkey. J Neurophysiol 35:915924.

Schwartz AB, Kettner RE, Georgopoulos AP (1988) Primate motor cortex and free arm movements to visual targets in three-dimensional space. I. Relations between single cell discharge and direction of movement. J Neurosci 8:2913-2927. 
Scott DW (1992) Multivariate density estimation. Theory, practice, and visualization. New York: Wiley.

Serruya MD, Hatsopoulos NG, Paninski L, Fellows MR, Donoghue JP (2002) Instant neural control of a movement signal. Nature 416:141-142.

Shadlen MN, Newsome WT (2001) Neural basis of a perceptual decision in the parietal cortex (area LIP) of the rhesus monkey. J Neurophysiol 86:1916-1936.

Smith PL, Ratcliff R (2004) Psychology and neurobiology of simple decisions. Trends Neurosci 27:161-168.

Sparks DL (1975) Response properties of eye movement-related neurons in the monkey superior colliculus. Brain Res 90:147-152.

Sparks DL (1978) Functional properties of neurons in the monkey superior colliculus: coupling of neuronal activity and saccade onset. Brain Res $156: 1-16$.

Sparks DL (1986) Translation of sensory signals into commands for control of saccadic eye movements: role of primate superior colliculus. Physiol Rev 66:118-171.

Spitzer H, Desimone R, Moran J (1988) Increased attention enhances both behavioral and neuronal performance. Science 240:338-340.

Sugrue LP, Corrado GS, Newsome WT (2005) Choosing the greater of two goods: neural currencies for valuation and decision-making. Nat Rev Neurosci 6:363-375.

Taylor DM, Tillery SIH, Schwartz AB (2002) Direct cortical control of 3D neuroprosthetic devices. Science 296:1829-1832.

Van Gisbergen JAM, Van Opstal AJ, Tax AAM (1987) Collicular ensemble coding of saccades based on vector summation. Neuroscience 21:541-555.

Wurtz RH, Goldberg ME (1972) Activity of superior colliculus in behaving monkey: III. Cells discharging before eye movements. J Neurophysiol 35:575-586. 